\title{
Gauge Theories on Four Dimensional Riemannian Manifolds
}

\author{
Thomas H. Parker*
}

Department of Mathematics, Harvard University, Cambridge, MA 02138, USA

\begin{abstract}
This paper develops the Riemannian geometry of classical gauge theories - Yang-Mills fields coupled with scalar and spinor fields - on compact four-dimensional manifolds. Some important properties of these fields are derived from elliptic theory: regularity, an "energy gap theorem", the manifold structure of the configuration space, and a bound for the supremum of the field in terms of the energy. It is then shown that finite energy solutions of the coupled field equations cannot have isolated singularities (this extends a theorem of K. Uhlenbeck).
\end{abstract}

\section{Introduction}

One of the major discoveries of physics in this century is the recognition that non-abelian Lie groups play a role in particle physics. For many years this was regarded as a peculiar aspect of quantum mechanics having no classical analogue. Then in 1954 C. N. Yang and R. Mills proposed a classical field theory incorporating these groups. Recently their theory has received considerable attention from both mathematicians and physicists.

Yang-Mills theory is easily described in terms of modern differential geometry. One begins with a principal bundle $P$ with compact Lie structure group $G$ over a manifold $M$. The Yang-Mills field is then the curvature $\Omega$ of a connection $V$ on $P$ which is a critical point of the action

$$
A(V)=\int_{M}|\Omega|^{2}
$$

When $G$ is the circle group the Yang-Mills field satisfies Maxwell's equations.

Physically, Yang-Mills fields represent forces. As such they interact with a second type of field - the field of a particle. This is interpreted as a section $\phi$ of a vector bundle associated to $P$ and the action for the system is essentially

$$
A(\nabla, \phi)=\int_{M}|\Omega|^{2}+|\nabla \phi|^{2}-m^{2}|\phi|^{2},
$$

* The author holds an A.M.S. Postdoctoral Fellowship 
where $m$ is a constant (the mass of the particle). The critical points of this action are solutions to a pair of coupled non-linear partial differential equations - the "coupled Yang-Mills equations." These are invariant under the infinite dimensional "gauge group" of all fibre preserving automorphisms of $P$. This setup constitutes a (classical) gauge theory and is the subject of this paper.

Four-dimensional compact Riemannian manifolds are the natural context for Yang-Mills theory for several reasons. First, the four-dimensional Yang-Mills action is bounded below by the characteristic number of the bundle, so the field is constrained by the topology. This is linked by invariant theory to the conformal invariance of the action. This conformal invariance occurs only in dimension four; it means that the relevant geometry lies in the conformal structure of the base manifold. The curvature is expressed in terms of the connection form $\omega$ by $\Omega=d \omega+\frac{1}{2}[\omega, \omega]$ and the Yang-Mills action is, essentially, $\int|d \omega|^{2}+|\omega|^{4}$. This is the sum of a gradient term $|d \omega|^{2}$ and a non-linear ("self-interaction") term $|\omega|^{4}$. By the Sobolev inequalities these terms are of compatible strength only in dimension four. Thus conformal invariance - which dictates the Sobolev inequalities - is reflected in the analytic aspects of Yang-Mills fields.

To date, the main analytic result for Yang-Mills fields is Uhlenbeck's proof [19] that a Yang-Mills field on a four-dimensional space with finite energy cannot have isolated singularities. As a consequence, a field on $\mathbb{R}^{4}$ with finite energy extends via stereographic projection to a field in a non-trivial bundle over $S^{4}$. This theorem is striking because it shows that the topology is inherent in the field; for example the quantity $\frac{1}{16 \pi^{2}} \int_{M} \Omega \wedge \Omega$ is always an integer - the characteristic number of the bundle. In this sense Uhlenbeck's theorem completes the circle: the analytic properties of the Yang-Mills field imply the topology.

It is natural to ask if isolated singularities can exist for coupled Yang-Mills fields. Our main result (Theorem 8.1) shows that such isolated singularities are indeed removable. The proof depends crucially on the conformal invariance of the coupled field equations.

In the first three sections we develop Yang-Mills theories on compact Riemannian four-manifolds. Section one is an overview of four-dimensional Riemannian geometry and is primarily intended to introduce the (considerable) notation used in subsequent sections. We begin by discussing the special features of the linear algebra of $\mathbb{R}^{4}$ which stem from the isomorphism $\operatorname{Spin}(4)=S U(2) \times S U(2)$. This algebraic structure carries over to vector bundles over four-manifolds and, when connections are introduced, leads to relationships between the curvature, topology and differential operators on these bundles.

In Sect. 2 we introduce the coupled Yang-Mills equations and show that the action is naturally associated to conformal structures on oriented four-manifolds. As in physics, we consider two types of equations: the "fermion" equations based on the Dirac operator for bundle-valued spinors, and the "boson" equations based on the bundle Laplacian. The key properties of the Yang-Mills equations - their gauge and conformal invariance - extend to these coupled equations.

The Yang-Mills equations are not elliptic because of gauge invariance. Section 4 contains a local slice theorem - similar to those of $[4,12,20]$ - for the action of the gauge group on the product of the space of connections and the space 
of fields. In Sect. 5 this slice theorem is used to construct local "gauges" (sections of the principal bundle). This breaks the gauge invariance of the equations, which are then elliptic and possess the expected regularity; for-example a bounded weak solution is $C^{\infty}$.

The last three sections are devoted to the proof of the removability of isolated singularities for finite energy solutions of the coupled field equations. This builds on the work of Uhlenbeck $[19,20]$. The proof involves three steps: (i) gauge independent estimates, (ii) a choice of gauge and the corresponding gauge dependent estimates, and (iii) an examination of how these estimates depend on the metric within the conformal class. Together, these yield an energy growth rate, from which the theorem follows.

The gauge independent estimate of Sect. 6 is perhaps of interest in other contexts: it shows that the supremum of the total field $F=\Omega+\nabla \phi+\phi$ is bounded by the $L^{2}$ norm (the energy) of $F$. One consequence of this is the fact that a solution to the coupled Yang-Mills equations is $O\left(\frac{1}{r^{2}}\right)$ around an isolated singularity. This growth rate is enough to establish the existence of a particularly nice gauge around the singularity using a theorem of Uhlenbeck. Estimates in this gauge are carried out in Sect. 7. These estimates go considerably beyond those of Uhlenbeck [19] by showing that the particle field $\phi$ satisfies an inequality (Theorem 7.6) analogous to Uhlenbeck's inequality on the curvature (Theorem 7.7).

The removability of singularities is proved in the last section. Note that this means that both the bundle and the field extend across the singularity. Finally, as an application, we prove an extension theorem: solutions of the coupled field equations $\mathbb{R}^{4}$ which decrease at infinity at a certain specified rate extend by stereographic projection to solutions over $S^{4}$.

\section{Four Dimensional Riemannian Geometry}

Riemannian geometry in dimension 4 is distinguished by the fact that the universal cover Spin(4) of the rotation group $\mathrm{SO}(4)$ is not a simple group, but decomposes as

$$
\operatorname{Spin}(4)=\mathrm{SU}(2) \times \mathrm{SU}(2) .
$$

On the group level this is seen by identifying $\mathbb{R}^{4}$ and $\mathbb{C}^{2}$ and with the quaternions $H$. We may regard $\mathrm{SU}(2)$ as the group of unit quaternions. For unit quaternions $g$ and $h$, the map $x \rightarrow g^{-1} x h$ is an orthogonal transformation of $H=\mathbb{R}^{4}$ with determinant 1 , and hence gives a homomorphism $\pi: \mathrm{SU}(2) \times \mathrm{SU}(2) \rightarrow \mathrm{SO}(4)$. This map has kernel $(-1,-1)$, so displays $\mathrm{SU}(2) \times \mathrm{SU}(2)$ as the 2 -fold universal covering group of $\mathrm{SO}(4)$.

On the algebra level the isomorphism $\underline{s o}(4)=\underline{s u}(2) \times \underline{\text { su}}(2)$ is a consequence of the Hodge star operation: ${ }^{*}: A^{2}\left(\mathbb{R}^{4}\right) \rightarrow \Lambda^{2}\left(\mathbb{R}^{4}\right)$ with $*^{2}=$ Identity, and the metric gives an identification $\underline{\mathrm{so}}(n)=\Lambda^{2}(V)$. Thus $\Lambda^{2}\left(\mathbb{R}^{4}\right)$ decomposes into \pm 1 eigenspaces: $\underline{\text { so }}(4)=\Lambda_{+}^{2} \oplus \Lambda_{-}^{2}$. The spaces $\Lambda_{ \pm}^{2}$ are 3-dimensional spaces of skewsymmetric matrices which are isomorphic as Lie algebras to $\underline{\mathrm{so}}(3)=\underline{\mathrm{su}}(2)$.

We will distinguish the two copies of SU(2) in Spin(4) by writing Spin(4) $=\mathrm{SU}_{+}(2) \times \mathrm{SU}_{-}(2)$ (this labeling is determined by orientation since a change in 
orientation switches $*$ to $-*$ ). The elements of $\Lambda_{+}^{2}\left(\Lambda_{-}^{2}\right)$ are called self-dual (anti self-dual) 2-forms.

The representations of Spin(4) can be determined using the isomorphism $\mathrm{Spin}(4)=\mathrm{SU}_{+}(2) \times \mathrm{SU}_{-}(2)$. First, recall the structure of the representation rings of SU(2). The fundamental representation $D_{1 / 2}$ is $\mathrm{SU}(2)$ acting on $\mathbb{C}^{2}$ in the usual manner and all other irreducible representations are symmetric powers $D_{k / 2}=\operatorname{Sym}^{k} D_{1 / 2}$ with $k \in \mathbb{Z}^{+}$. Tensor products of these representations decompose according to the Clebsch-Gordan formula

$$
D_{k / 2} \otimes D_{l / 2}=D_{k+l / 2} \oplus D_{k+l-2 / 2} \oplus \ldots \oplus D_{|k-l| / 2} .
$$

The representation ring of $\operatorname{Spin}(4)$ is generated by the two fundamental representations - the spin representations $-D_{1 / 2}^{ \pm}$obtained by projecting Spin(4) onto $\mathrm{SU}_{ \pm}(2)$ and applying $D_{1 / 2}$. The representation $D_{k / 2}^{+} \otimes D_{1 / 2}^{-}$has dimension $(k+1)(l+1)$ and factors through $\mathrm{SO}(4)$ if and only if $k+l$ is even; these are the orthogonal irreducible representations of Spin(4) and all others are symplectic.

It is often convenient to view the spin representations in the context of Clifford algebras. For details we refer to Atiyah et al. [3].

Let $E=\mathbb{R}^{2 k}$ with the positive definite inner product $g$ and let $\left\{e_{i}\right\}$ be an orthonormal basis of $E$. The Clifford algebra $C(E)$ of $E$ and $g$ is the graded algebra $C(E)=T(E) / I$, where $T(E)$ is the tensor algebra on $E$ and $I$ is the two-sided ideal generated by the elements $x \otimes x+g(x, x) \cdot 1$ for $x \in E$. Thus $C(E)$ is generated by $\left\{e_{i_{1}} \ldots e_{i_{r}} \mid i_{1}<i_{2}<\ldots<i_{r}, r \leqq 2 k\right\}$ with relations $e_{i} e_{j}+e_{j} e_{i}=-2 \delta_{i j}$. The map $e_{i_{1}} \wedge \ldots \wedge e_{i_{r}} \rightarrow e_{i_{1}} \ldots e_{i_{r}}$ from exterior algebra on $E$ gives a (non-canonical) isomorphism $A^{*}(E) \simeq C(E)$ of graded vector spaces. Furthermore, the complexified Clifford algebra $C_{c}(E)$ is algebra isomorphic to the endomorphisms of a $2^{k}$-dimensional complex vector space $V: A_{c}^{*}(E) \simeq C_{c}(E) \simeq$ End $(V)$.

The group $\mathrm{SO}(2 k)$ acts on $E$ and this action extends, showing that $A^{*}(E) \simeq C(E)$ as $\mathrm{SO}(2 k)$ modules. In fact, $\mathrm{SO}(2 k)$ acts on $C(E)$ by inner automorphisms and this gives an embedding of $\operatorname{Spin}(2 k)$ in $C^{\text {even }}(E)$ with group multiplication given by multiplication in the Clifford algebra. On the algebra level $\underline{\operatorname{so}}(2 k)=A^{2}(E) \rightarrow C^{2}(E)$ embeds as the tangent space to $\operatorname{Spin}(2 k)$ at the identity and the restriction of Clifford multiplication $A^{2}(E) \otimes A^{2}(E) \rightarrow C(E)$ coincides with the Lie bracket under the identification $A^{2}(E)=\underline{\mathrm{so}}(2 k)$ by $\left(e^{i} \otimes e_{j}-e^{j} \otimes e_{i}\right) \mapsto-\frac{1}{2} e^{i} \cdot e^{j}$.

The inclusion $\operatorname{Spin}(2 k) C C_{c}(E) \approx$ End $(V)$ makes $V$ a Spin $(2 k)$ representation. Since $2 k$ is even this is a reducible representation : $\varepsilon=e_{1} e_{2} \ldots e_{2 k}$ is invariant, $\varepsilon^{2}=1$, and hence $V=V_{+} \oplus V_{-}$decomposes into the \pm 1 eigenspaces of $\varepsilon$. The even elements of $C(E)$ commute with $\varepsilon$, so $C^{\text {even }}(V)=\operatorname{End}\left(V_{+}\right) \oplus \operatorname{End}\left(V_{-}\right)$as $\operatorname{Spin}(2 k)$ modules. The odd elements of $C(E)$ interchange $V_{+}$and $V_{-}$. In particular, each $x \in E$ maps $V_{+} \rightarrow V_{-}$and $V_{-} \rightarrow V_{+}$, giving the Clifford multiplication map $E \otimes V_{ \pm} \rightarrow V_{\mp}$ which we denote by $x \otimes \phi \mapsto x \cdot \phi$.

In four dimensions the representations $V_{ \pm}$are the 2-dimensional complex spin representations $D_{1 / 2}^{ \pm}$mentioned above. In the isomorphism $A_{c}^{*}\left(\mathbb{R}^{4}\right) \simeq C_{c}\left(\mathbb{R}^{4}\right) \simeq \operatorname{End}(V), A_{c}^{1} \simeq \operatorname{Hom}\left(V_{+}, V_{-}\right) \simeq \operatorname{Hom}\left(V_{-}, V_{+}\right) \simeq A_{c}^{3}$ with the real parts embedded in $\operatorname{Hom}\left(V_{+}, V_{-}\right) \otimes \operatorname{Hom}\left(V_{-}, V_{+}\right)$as $A^{1}=\left\{\left(A,-A^{*}\right)\right\}$. Also, $A_{c}^{2}$ decomposes as $\Lambda_{+c}^{2} \oplus \Lambda_{-c}^{2}$ with $\Lambda_{ \pm c}^{2}=\left[\operatorname{Hom}\left(V_{ \pm}, V_{ \pm}\right)\right]^{0}$, where ${ }^{0}$ denotes the component consisting of traceless matrices. The real parts $\Lambda_{ \pm}^{2}$ consist of traceless skew hermitian endomorphisms of $V_{ \pm}$. 
These algebraic facts carry over to vector bundles on oriented four-manifolds except for one topological obstruction. By studying the fibration of classifying spaces corresponding to the sequence $0 \rightarrow \mathbb{Z}_{2} \rightarrow \mathrm{Spin}(4) \rightarrow \mathrm{SO}(4) \rightarrow 0$ and noting that $B \mathbb{Z}_{2}=K\left(\mathbb{Z}_{2}, 1\right)$, one sees that an oriented manifold $M^{4}$ has a spin structure if and only if its second Stiefel-Whitney class $\omega_{2} M$ vanishes, and that when $\omega_{2} M=0$ the spin structures are classified by $H^{1}\left(M ; \mathbb{Z}_{2}\right)$.

When $M$ has a spin structure the frame bundle $F$ of $T^{*} M$ lifts to a Spin(4) bundle and there is an associated vector bundle

$$
E_{k, l}=F \times_{(k, l)}\left(D_{k / 2}^{+} \otimes D_{l / 2}^{-}\right)
$$

for each irreducible representation of Spin(4). In particular, the spin bundles $V_{+}=E_{1,0}$ and $V_{-}=E_{0,1}$ and the total spin bundle $V=V_{+} \oplus V_{-}$are defined. One can also form the Clifford algebra bundle $C\left(T^{*} M\right)$ on the cotangent space and obtain a global Clifford multiplication map

$$
\Gamma\left(T^{*} M\right) \otimes \Gamma\left(V_{ \pm}\right) \rightarrow \Gamma\left(V_{\mp}\right) .
$$

Sections of $V$ are called spinor fields, or simply spinors.

When $\omega_{2} M \neq 0$ the only bundles $E_{k, l}$ which are globally defined are those associated to a representation which factors through SO(4) - those with $k+l$ even.

\section{Connections, Curvature, and Operators}

Now assume that $M$ is a Riemannian manifold. The metric determines the LeviCivita covariant derivative

$$
\nabla: \Gamma\left(T^{*} M\right) \rightarrow \Gamma\left(T^{*} M \otimes T^{*} M\right)
$$

on the cotangent space. Choosing a local basis of sections $\left\{e^{i}\right\}$ of $T^{*} M$ we can write $\nabla e^{i}=\sum \omega_{k}^{i} \otimes e^{k}$, where $\left\{\omega_{k}^{i}\right\}$ are the connection 1-forms. The nature of these connection forms is best seen in the context of connections on an arbitrary bundle.

Let $G$ be a compact semisimple Lie group with Lie algebra $g$ and let $\pi: P \rightarrow M$ be a principal $G$-bundle over manifold $M$. A connection on $P$ is a choice of an equivariant horizontal subspace on $T_{*} P$, or dually a $g$-valued 1 -form on $P$ which (i) has horizontal kernel: $\omega\left(i_{*} A\right)=A$ for $A \in g$, where $i_{*}: g \rightarrow T_{*} P$ is the natural inclusion into the vertical subspace, and (ii) is equivariant: $g^{*} \omega(X)=\left(\mathrm{Ad}^{-1}\right) \omega(X)$ for $x \in \Gamma\left(T_{*} P\right)$ and $g \in G$.

Let $\mathscr{C}$ denote the affine space of $C^{\infty}$ connections of $P ; \mathscr{C}$ becomes a vector space when a "base" connection $\omega_{0}$ is fixed. The equivariance property shows that the difference $\eta=\omega-\omega_{0}$ pulls down to $M$ as a 1 -form with values in the adjoint bundle $P \times_{\text {Ad } \mathscr{F}}$, which we shall also denote by $\mathscr{g}$. As such, it determines a covariant derivative map

$$
\nabla: \Gamma(g) \rightarrow \Gamma\left(g \otimes T^{*} M\right)
$$

by $\phi \mapsto \nabla_{0}+[\eta, \phi]$, where $\nabla_{0}$ is the covariant derivative corresponding to $\omega_{0}$. If $\varrho: G \rightarrow \operatorname{Aut}(\bar{E})$ is a representation and $E=P \times{ }_{e} \bar{E}$ the associated vector bundle, then $\omega$ induces a covariant derivative

$$
\nabla^{E}: \Gamma(E) \rightarrow \Gamma\left(E \otimes T^{*} M\right)
$$

on $E$ by applying the Lie algebra representation $\varrho: g \rightarrow \operatorname{End}(\bar{E})$ to $(1.3)$. 
For example, if $P$ is the frame bundle of $T^{*} M$ the Riemannian connection can be described either in terms of the covariant derivative (1.2) or in terms of the corresponding so(n)-valued connection for $\omega$. In a local frame $\omega=\left\{\omega_{k}^{i}\right\}$ are the forms described above.

Given a connection $\nabla^{E}$ on a vector bundle $E$ we can form several natural operators from $\nabla^{E}$ and a symbol map. Extending $V^{E}$ to the covariant derivative $\mid \nabla=V \otimes 1+1 \otimes V^{E}$ on $A^{*} \otimes E$ (where $V$ is the Riemannian connection on $A^{*}$ ) and taking exterior multiplication or its adjoint (contraction) as the symbol, we obtain an exterior differentiation $D: \Gamma\left(\Lambda^{*} \otimes E\right) \rightarrow \Gamma\left(\Lambda^{*+1} \otimes E\right)$ and its formal adjoint $D^{*}$. In a local orthonormal frame $\left\{e^{i}\right\}$

$$
\left\{\begin{array}{l}
D \phi=\sum e^{i} \wedge \nabla_{i} \phi \\
\left.D^{*} \phi=-\sum e_{i}\right\lrcorner \nabla \phi
\end{array} \quad \phi \in \Gamma\left(\Lambda^{*} \otimes E\right) .\right.
$$

There are also two second order operators of interest: the trace Laplacian

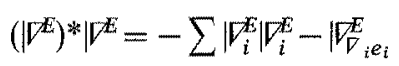

on $\Gamma(E)$ and the bundle Laplace-Beltrami operator $\square=\left(D D^{*}+D^{*} D\right)$ on $\Gamma\left(\Lambda^{*} \otimes E\right)$. We will discuss these later in relation to the Weitzenböck formulae.

The covariant derivative $\nabla=\nabla^{\mathscr{y}}$ of (1.3) now extends via (1.5) to an exterior differentiation $D$ on the space of sections $A^{*}=\Gamma\left(A^{*} \otimes g\right)$

$$
\Gamma(g)=A^{0} \stackrel{\nabla}{\longrightarrow} A^{1} \stackrel{D}{\longrightarrow} A^{2} \stackrel{D}{\longrightarrow} \ldots
$$

by $\phi \mapsto \nabla_{0}+[\eta, \phi]$, where $\nabla_{0}$ is the covariant derivative corresponding to $\omega_{0}$. Then $A^{*}$ is a graded Lie algebra with operations

(i) The bracket on $g$ and exterior multiplication give a map $A^{p} \otimes A^{q} \rightarrow A^{p+q}$ which we denote by $\omega \otimes \eta \mapsto[\omega, \eta]$.

(ii) The Killing form gives a positive definite metric on $g$ and a multiplication

by

$$
A^{p} \otimes A^{q} \rightarrow \Gamma\left(A^{p+q}\left(T^{*} M\right)\right)
$$

$$
(\alpha \otimes A) \otimes(\beta \otimes B) \mapsto\langle A, B\rangle \alpha \wedge \beta
$$

for $\alpha \in \Gamma\left(A^{p}\right), \beta \in \Gamma\left(\Lambda^{q}\right)$, and $A, B \in \Gamma(g)$. We denote this by $\omega \otimes \eta \vdash \omega \wedge \eta$. These operations satisfy (cf. Atiyah and Bott [1])

(a) $\left[\omega^{p}, \omega^{q}\right]=(-1)^{p q+1}\left[\omega^{q}, \omega^{p}\right]$,

(b) $\omega^{p} \wedge \omega^{q}=(-1)^{p q} \omega^{p} \wedge \omega^{p}$,

(c) $(-1)^{p r}\left[\omega^{p},\left[\omega^{q}, \omega^{r}\right]\right]+(-1)^{p q}\left[\omega^{q},\left[\omega^{r}, \omega^{p}\right]\right]+(-1)^{q r}\left[\omega^{r},\left[\omega^{p}, \omega^{q}\right]\right]=0$ (Jacobi Identity),

(d) $[\omega, \eta] \wedge \xi=\omega \wedge[\eta, \xi]$ (Invariance of the Killing form),

(e) $D\left[\omega^{p}, \eta\right]=\left[D \omega^{p}, \eta\right]+(-1)^{p}\left[\omega^{p}, D \eta\right]$,

(f) $d\left(\omega^{p} \wedge \eta\right)=D \omega^{p} \wedge \eta+(-1)^{p} \omega^{p} \wedge D \eta$.

The curvature of a connection $\omega$ on the principal bundle $P$ is the $g$-valued 2-form $\Omega(X, Y)=d \omega(h X, h Y)$, where $h$ is the projection onto the horizontal subspace of $\omega$. In fact, $D=d \circ h$ is a derivation on equivariant $g$-valued 1 -forms on $P$ given by $D \phi=d \phi+[\omega, \phi]$ for 1 -forms with vertical kernel and $D \phi=d \phi$ $+\frac{1}{2}[\omega, \phi]$ for connection forms $\phi$ (Kobayashi and Nomizu, Sect. 2.5). In particular $\Omega=d \omega+\frac{1}{2}[\omega, \omega]$ on $P$. Now fix a connection $\omega_{0}$. For any other connection $\omega$, the 
difference $\eta=\omega-\omega_{0}$ descends to $M$ as an element of $A^{1}$ and the difference of the curvatures is

$$
\begin{aligned}
\Omega-\Omega_{0} & =d \eta+\frac{1}{2}[\omega, \omega]-\frac{1}{2}\left[\omega_{0}, \omega_{0}\right]=d \eta+\frac{1}{2}[\eta, \eta]+[\omega, \eta], \\
\Omega & =\Omega_{0}+D_{0} \eta+\frac{1}{2}[\eta, \eta] .
\end{aligned}
$$

There is a second way to describe $\Omega$ in $A^{*}$. Any $\phi \in A^{\circ}$ lifts to an equivariant $g$-valued function on $P$ and $D \phi$ has vertical kernel. Hence

$$
\begin{aligned}
D \circ D(\phi) & =d(D \phi)+[\omega, D \phi]=d(d \phi+[\omega, \phi])+[\omega, D \phi] \\
& =[D \omega, \phi]-[\omega, D \phi]+[\omega, D \phi]=[\Omega, \phi] .
\end{aligned}
$$

This formula descends to the base:

$$
D \circ \nabla(\phi)=[\Omega, \phi] \text { for } \phi \in A^{0},
$$

i.e., the curvature endomorphism $\Omega$ on $A^{0}$ is the composition of the first two operators of (1.6). Similarly, the curvature of an associated bundle $E$ is $\Omega^{E} \in \Gamma\left(\Lambda^{2} \otimes\right.$ End $\left.(E)\right)$ defined either by $\Omega^{E}=\varrho(\Omega)$ where $\varrho: g \rightarrow$ End $(\bar{E})$ is the defining representation, or as the composition $\Omega^{E}=D^{E} \circ \nabla^{E}$ in the sequence

$$
\Gamma(E) \stackrel{\nabla^{E}}{\longrightarrow} \Gamma\left(\Lambda^{\prime} \otimes E\right) \stackrel{D^{E}}{\longrightarrow} \Gamma\left(\Lambda^{2} \otimes E\right) \stackrel{D^{E}}{\longrightarrow} \ldots
$$

corresponding to (1.6). In terms of a local basis of vector fields $\left\{e_{i}\right\}$ and dual forms $\left\{e^{i}\right\}$

$$
\Omega(\phi)=D \circ \nabla \phi=D\left(\sum_{i} \nabla_{i} \phi e^{i}\right)=\sum_{i_{s}, k}\left(\nabla_{i} \phi e^{k} \wedge \nabla_{k} e^{i}+\nabla_{k} \nabla_{i} \phi e^{k} \wedge e^{i}\right),
$$

and noting that $\nabla_{i} e^{j}-\nabla_{j} e^{i}=\left[e^{i}, e^{j}\right]$ for the Riemannian connection,

$$
\Omega_{i j}=\nabla_{i} \nabla_{j}-\nabla_{j} \nabla_{i}-\nabla_{\left[e_{i}, e_{j}\right]}
$$

and similarly for $\Omega^{E}$.

An important consequence of the Jacobi identity is the Bianchi identity $D \Omega=0$.

When $P$ is the frame bundle of $M$ it has a metric connection and we will denote the Riemannian curvature of $M$ by $R \in \Gamma\left(\Lambda^{2} \otimes \underline{s o}(n)\right)$.

In four dimensions the Riemannian curvature $R \in \Lambda^{2} \otimes \underline{s o}(4)=\Lambda^{2} \otimes \Lambda^{2}$ decomposes under the splitting $\Lambda^{2}=\Lambda_{+}^{2} \oplus \Lambda_{-}^{2}$. In fact, because of the symmetry $R_{i j k l}=R_{k l i j}, R$ is an element of the symmetric tensor product $\operatorname{Sym}^{2}\left(\Lambda_{+}^{2} \oplus \Lambda_{-}^{2}\right)$. As a $\mathrm{Spin}(4)=\mathrm{SU}{ }_{+}(2) \times \mathrm{SU} \mathrm{U}_{-}(2)$ module this breaks into 5 irreducible pieces according to the Clebsh-Gordon formula

$$
\left(\operatorname{Sym}^{2} \Lambda_{+}^{2}\right)^{0} \oplus 1 \oplus\left[\Lambda_{+}^{2} \otimes \Lambda_{-}^{2}\right] \oplus\left(\operatorname{Sym}^{2} \Lambda_{-}^{2}\right)^{0} \oplus 1,
$$

where ${ }^{0}$ denotes the traceless elements in the symmetric product. The components of $R$ under this decomposition are $R=\left(W^{+}, \frac{s}{12}, 2 B, W^{-m}, \frac{s}{12}\right)$ where $s$ is the scalar curvature, $B$ is the traceless Ricci tensor, and $W^{ \pm}$are the self-dual and anti-selfdual components of the conformally invariant Weyl tensor (cf. Singer and Thorpe). This decomposition distinguishes several important classes of four-manifolds : $M^{4}$ is Einstein if $B \equiv 0$, conformally flat if $W \equiv 0$, and self-dual (anti-self-dual) if $W^{-} \equiv 0\left(W^{+} \equiv 0\right)$. 
On a four dimensional Riemannian manifold the metric covariant derivative on the spin bundle $V_{+}$is a map $\nabla: \Gamma\left(V_{+}\right) \rightarrow \Gamma\left(V_{+} \otimes T^{*} M\right)$. Note that $V_{+} \otimes T_{c}^{*} M \simeq V_{+} \otimes V_{+}^{*} \otimes V_{-}=V_{-} \oplus A_{+c}^{2}$ and that the projection $V_{+} \otimes T^{*} M \rightarrow V_{-}$is Clifford multiplication. Thus $V$ on $V_{ \pm}$decomposes into two operators: the Dirac Operator $\mathscr{B}: \Gamma\left(V_{+}\right) \rightarrow \Gamma\left(V_{\mp}\right)$, whose symbol is Clifford multiplication, and the Twister Operator $\overline{\mathscr{O}}: \Gamma\left(V_{ \pm}\right) \rightarrow \Gamma\left(\Lambda_{+}^{2}\right)$, whose symbol is the orthogonal complement of Clifford multiplication. In a local orthonormal frame $\left\{e^{i}\right\}$

$$
\left\{\begin{array}{l}
\mathscr{D} \phi=\sum e^{i} \cdot \nabla_{i} \phi \\
\overline{\mathscr{D}} \phi=\nabla \phi+\frac{1}{4} \sum e^{i} \cdot \mathscr{D} \phi \otimes e^{i} \quad \phi \in \Gamma(V)
\end{array}\right.
$$

The Dirac operator is elliptic and is formally self-adjoint on the total spin bundle $V$.

The metric connection also gives a covariant derivative on each bundle $E_{k, t}$ associated to the frame bundle of $T^{*} M, \nabla: \Gamma\left(E_{k, l}\right) \rightarrow \Gamma\left(E_{k, l} \otimes T^{*} M\right)$. Because

$$
E_{k, l} \otimes T^{*} M \simeq E_{k, l} \otimes E_{1,1} \approx E_{k+1, l+1} \oplus E_{k+1, l-1} \oplus E_{k-1, l+1} \oplus E_{k-1, l-1}
$$

$\nabla$ decomposes into four operators : two Dirac operators, a Twistor operator, and the adjoint of this Twistor operator. For example, $d \oplus d^{*}: \Lambda_{c}^{1} \oplus \Lambda_{1}^{3} \rightarrow \Lambda_{c}^{2}$ $=A_{+c}^{2} \oplus A_{-c}^{2}$ is a homogeneous first order operator $E_{1,1} \rightarrow E_{2,0} \oplus E_{0,2}$ which coincides with the sum of the two Dirac operators on $E_{1,1}$.

Finally, if $P$ is a principal bundle with connection over $M^{4}$ and $E$ is a vector bundle associated to $P$, then the covariant derivative on $E$ extends to $\mid \nabla=\nabla \otimes 1$ $+1 \otimes \nabla^{E}$ on $E_{k, l} \otimes E$ and we obtain Dirac and Twistor operators on $E_{k, l} \otimes E$.

\section{Topological Invariants}

Compact four dimensional manifolds $M$ possess two real characteristic classes: the Pontryagin class $p_{1} M$ and the Euler class $e(M)$. By Chern-Weil theory these can be expressed locally as polynomials in the curvature of $M$ and hence as polynomials in the irreducible components $\left\{s, B, W^{ \pm}\right\}$of the curvature

$$
\begin{aligned}
& p_{1} M=\frac{1}{4 \pi^{2}} \int_{M}\left|W^{+}\right|^{2}-\left|W^{-}\right|^{2}, \\
& \chi(M)=\frac{1}{8 \pi^{2}} \int_{M} \frac{s^{2}}{24}-2|B|^{2}+\left|W^{+}\right|^{2}+\left|W^{-}\right|^{2} .
\end{aligned}
$$

More generally, let $G$ be compact simple Lie group. Then $H^{i}(B G ; \mathbb{R})$ vanishes for $i=1,2,3$ and is $\mathbb{R}$ for $i=4$. Thus there is a single real characteristic class for principal $G$-bundles over $M^{4}$; it lies in dimension 4. In the context of Yang-Mills theory the corresponding characteristic number is called the Pontryagin Index of the bundle and is denoted by $k$. According to the Chern-Weil prescription it is obtained by substituting the curvature $\Omega$ of $P$ - or of the adjoint bundle $g$ - into the Killing form. In terms of the anti-self-dual components $\Omega^{ \pm}$of $\Omega$

$$
k=\frac{1}{8 \pi^{2}} \int_{M}\left|\Omega^{+}\right|^{2}-\left|\Omega^{-}\right|^{2} .
$$




\section{Weitzenböck Formulae}

Let $M$ be a spin four-manifold with Riemannian connection $\nabla$ and curvature $R$ and let $E$ be a vector bundle over $M$ with connection $\nabla^{E}$ and curvature $\Omega^{E}$. Then the Dirac operator

$$
\mathscr{D}: \Gamma(V \otimes E) \rightarrow \Gamma(V \otimes E)
$$

is defined for $E$-valued spinors by $\mathscr{D}=\sum e^{i} \cdot \nabla_{i}$, where $\nabla=\nabla \otimes 1+1 \otimes V^{E}$ is the total covariant derivative on $V \otimes E$. The square of this operator has an algebraic decomposition into Laplacian and curvature terms; such a decomposition is called a Weitzenböck formula.

To compute $\mathscr{D}^{2}$ it is convenient to choose an orthonormal local frame $\left\{e^{i}\right\}$ around a point $x \in M$ and vector fields $\left\{e_{i}\right\}$ dual to $\left\{e^{i}\right\}$ with $\left(\nabla_{e_{i}} e^{j}\right)_{x}=0$ for all $i, j$. Since $\nabla$ has no torsion it follows that $\left[e_{i}, e_{j}\right]_{x}=0$ and that the total curvature $\Omega=R \otimes 1+1 \otimes \Omega^{E}$ of $V \otimes E$ is $\Omega_{i j}=\left|\nabla_{1}\right| \nabla_{j}-\nabla_{j} \nabla_{i}$. Squaring $\mathscr{D}$ and separating the symmetric and skew-symmetric parts:

or

$$
\begin{aligned}
\mathscr{D}^{2} & =\left(\sum e^{i} \cdot \mid \nabla_{j}\right)\left(\sum e^{j} \cdot \mid \nabla_{j}\right)=\sum e^{i} \cdot e^{j} \cdot\left|\nabla_{i}\right| \nabla_{j} \\
& =-\sum \mid \nabla_{j} \nabla_{i}+\sum_{i, j} e^{i} \cdot e^{j} \cdot\left(\left|\nabla_{i}\right| \nabla_{j}-\mid \nabla_{j} \nabla_{j}\right)
\end{aligned}
$$

$$
\mathscr{D}^{2}=\left|\nabla^{*}\right| \nabla+\frac{1}{2} \sum_{i, j} e^{i} \cdot e^{j} \cdot R_{i j} \otimes 1+\frac{1}{2} \sum_{i, j} e^{i} \cdot e^{j} \cdot\left(1 \otimes \Omega_{i j}^{E}\right) .
$$

The principal term $\left|\nabla^{*}\right| \nabla$ is the positive trace Laplacian of $\mid \nabla$. The endomorphism $\mathscr{R}=\frac{1}{2} \sum e^{i} \cdot e^{j} \cdot R_{i j}$ of $V$ involves only the Riemannian curvature of $V$ and can be expressed in terms of the irreducible components $\left\{s, B, W^{ \pm}\right\}$of $R$ as follows.

$R$ acts on spinors via the spin representation $e^{k} \wedge e^{l} \mapsto-\frac{1}{2} e^{k} \cdot e^{l}$. and the image $c(R)=-\frac{1}{8} \sum R_{i j k l} e^{i} \cdot e^{j} \cdot e^{k} \cdot e^{l}$ is an even element of $C\left(\Lambda^{1}\right)$, so has three components $\Lambda^{0} \oplus \Lambda^{2} \oplus \Lambda^{4}$ according to whether 2 pair $/ 1$ pair/none of the indices of $e^{i} \cdot e^{j} \cdot e^{k} \cdot e^{l}$ are the same. But the $\Lambda^{2}$ component vanishes because $R: A^{2} \rightarrow \Lambda^{2}$ is symmetric, the $A^{4}$ component vanishes by the Bianchi identity, and the $A^{0}$ component is

$$
-\frac{1}{8} \sum\left(R_{i j i j} e^{i} \cdot e^{j} \cdot e^{i} \cdot e^{j}+R_{i j j i} e^{i} \cdot e^{j} \cdot e^{j} \cdot e^{i}\right)=\frac{s}{4} .
$$

Thus we arrive at our first Weitzenböck formula: the square of the Dirac operator on $E$-valued spinors is

$$
\mathscr{D}^{2}=\left|\nabla^{*}\right| \nabla+\frac{s}{4}+\frac{1}{2} \sum e^{i} \cdot e^{j} \cdot \Omega_{i j}^{E} .
$$

By replacing $E$ by $V^{*} \otimes E$ we get a Dirac operator on $V \otimes V^{*} \otimes E \simeq A^{*} \otimes E$, i.e. on $E$-valued forms. For this $\mathscr{D}$

$$
\mathscr{D}^{2}=|\nabla *| \nabla+\frac{1}{2} \sum e^{i} \cdot e^{j} \cdot R_{i j}^{A^{*}}+\frac{1}{2} \sum e^{i} \cdot e^{j} \cdot \Omega_{i j}^{E}
$$

and $\mathscr{D}^{2}$ is closely related to the bundle Laplace-Beltrami operator $\square=D D^{*}$ $+D^{*} D$. Indeed, $\mathscr{D}=D+D^{*}$ on $A^{*} \otimes E$ and $\mathscr{D}^{2}=\square+D^{2}+\left(D^{*}\right)^{2}$. Although $D^{2}$ and $\left(D^{*}\right)^{2}$ are not zero, we can ignore them by focusing only on that component of $\mathscr{D}^{2}$ which preserves degree and then (1.15) decomposes 
Under the isomorphism $V \otimes V^{*}=\Lambda^{*}, \quad e_{i} \cdot e_{j} \cdot$ corresponds to $\left.\left.-\left(e_{i}\right\lrcorner e^{j} \wedge \cdot+e^{i} \wedge e_{j}\right\lrcorner \cdot\right)$, so the middle term of $(1.15)$ is

$$
\left.\left.\mathscr{R}=-\frac{1}{2} \sum R_{i j}^{\Lambda^{*}}\left(e_{i}\right\lrcorner e^{j} \wedge \cdot+e^{i} \wedge e_{j}\right\lrcorner \cdot\right) .
$$

Alternatively, we can write $R^{A^{*}}=R^{V} \otimes 1+1 \otimes R^{V^{*}}$ and

$$
\mathscr{R}=-\frac{1}{8} \sum_{i j k l} R_{i j k l} e^{i} \cdot e^{j} \cdot e^{k} \cdot e^{l} \cdot \otimes 1+\frac{1}{2} \sum_{i<j, k<l} R_{i j k l} e^{i} \cdot e^{j} \cdot \otimes e^{k} \cdot e^{l} \cdot
$$

where the first term is $\frac{s}{4}$ as above. In the second term $R$ is acting through the Clifford multiplication map

$$
R \in \Lambda^{2} \otimes\left(\Lambda^{2}\right)^{*} \underset{c \otimes c}{\longrightarrow} \operatorname{End}(V) \otimes \operatorname{End}\left(V^{*}\right) \rightarrow \operatorname{End}\left(\Lambda^{*}\right)
$$

which can be computed on each component of $A^{*}$ using the decomposition (1.10) of the curvature.

1. Scalars embed in $A^{*}=V \otimes V^{*}$ as multiples of the identity and on them

$$
\left.\left.\left(e_{i}\right\lrcorner e^{j} \wedge \cdot+e^{i} \wedge e_{j}\right\lrcorner\right)=\delta_{i j} .
$$

Hence the Weitzenböck formula for $E$-valued scalars is simply

$$
\square=\left|\nabla^{*}\right| \nabla .
$$

2. One-forms embed in $V \otimes V^{*}$ as skew adjoint elements of $\left(V_{+} \otimes V_{-}^{*}\right) \oplus\left(V_{-} \otimes V_{+}^{*}\right)$ and the only traceless Ricci tensor can act on them through (1.16). Thus $\mathscr{R}=\frac{s}{4}+B()=\operatorname{Ric}()$ is contraction with the Ricci tensor (more explicitly,

$$
\left.\left.\mathscr{R}=-\frac{1}{2} \sum_{k<l} R_{i j k l}\left(e_{i}\right\lrcorner e^{i} \wedge \cdot+e^{i} \wedge e_{j}\right\lrcorner \cdot\right)\left(e^{k} \otimes e_{l}-e^{l} \otimes e_{k}\right)
$$

applied to a 1-form $e^{m}$ is $\left.\mathscr{R}\left(e^{m}\right)=\sum R_{i m} e^{i}\right)$. The Weitzenböck formula for 1-forms is

$$
\square=\left|\nabla^{*}\right| \nabla+\operatorname{Ric}()+\frac{1}{2} \sum e^{i} \cdot e^{j} \cdot \Omega_{i j}^{E} .
$$

3. Self-dual 2-forms embed in $V \otimes V^{*}$ as traceless self-adjoint elements and only the scalar and self-dual Weyl curvatures can act on them. In fact, (1.16) restricted to $\operatorname{End}\left(A_{+}^{2}\right)$ is twice the identity and, by $(1.10), \mathscr{R}=\frac{s}{4}+\frac{s}{12}+W^{+}()$. As noted earlier, Clifford multiplication coincides with brackets in $\underline{\underline{s}}(4)=\Lambda^{2}$, so for

$$
\begin{aligned}
\phi & =\sum \phi_{k l} e^{k} \wedge e^{l} \in \Gamma\left(\Lambda_{+}^{2} \otimes E\right), \\
\frac{1}{2} \sum e^{i} \cdot e^{j} \cdot \Omega_{i j}^{E}(\phi) & =-\left[\left(\Omega^{E}\right)^{+}, \phi\right]=\left[\Omega_{j k}\left(\phi_{i j}\right)-\Omega_{i j}\left(\phi_{j k}\right)\right] \quad e^{i} \wedge e^{k} .
\end{aligned}
$$

The Weitzenböck formula is

$$
\square=\nabla^{*} \mid \nabla+\frac{s}{3}+W^{+}()-\left[\left(\Omega^{\bar{E}}\right)^{+}, \cdot\right] .
$$


The same formula holds for anti-self-dual 2-forms when $W^{+}$and $\left(\Omega^{E}\right)^{+}$are replaced by $W^{-}$and $\left(\Omega^{E}\right)^{-}$.

4. Since $\square$ commutes with the star operator, the Weitzenböck formula on $E$-valued 4-forms is given by (1.17) and on $E$-valued 3-forms by (1.18) with the curvature terms replaced by their *-adjoints.

\section{Sobolev Spaces}

For functions on a bounded domain $D$ in $\mathbb{R}^{n}$ the Sobolev space $L_{k, p}(D)$ is the completion of the space of $C^{\infty}$ functions in the norm

$$
\|f\|_{k, p}=\left(\int \sum_{D|\alpha|=1}^{k}\left|\partial_{\alpha} f\right|^{p}\right)^{1 / p}
$$

These spaces are related by the Sobolev embedding theorems: for $p, q \geqq 1$, the inclusion $L_{k, p}(D) \rightarrow L_{l, q}(D)$ is continuous for $k-\frac{n}{p} \geqq l-\frac{n}{q}$ and compact for $k-\frac{n}{p}$ $>l-\frac{n}{q}$, and the inclusion $C^{l}(D) \rightarrow L_{k, p}(D)$ is compact for $k-\frac{n}{p}>l$. This setup carries over to vector bundles on compact Riemannian manifolds $M$ (Palais). In fact, given a $C^{\infty}$ vector bundle $E$ with metric over $M$, we can complete the space $\Gamma^{\infty}(E)$ of $C^{\infty}$ sections of $E$ by either

(i) choosing a coordinate covering $\left\{V_{i}\right\}$ and a subordinate partition of unity $\left\{\phi_{i}\right\}$ and defining $L_{k, p}(E)$ by the inclusion $\left\{\phi_{i}\right\}: \Gamma_{M}^{\infty}(E) \rightarrow \oplus_{i} \Gamma_{U_{i}}^{\infty}(E)$;

(ii) choosing a connection $V$ on $E$ compatible with the metric and defining the norm \|\|$_{k, p, V}$ by the above formula with $\partial$ replaced by $\nabla$.

These procedures yield equivalent Banach spaces. We will use the second definition, and, after fixing a connection, write $\|_{k, p, \nabla}$ as $\|_{k, p}$.

In dimension 4 the relevant Sobolev inequality for functions is

$$
\left(\int_{B_{r}}|f|^{4}\right)^{1 / 2} \leqq c\left[\int_{B_{r}}|d f|^{2}+r^{-2} \int_{B_{r}}|f|^{2}\right]
$$

on the ball $B_{r}$ of radius $r$, or

$$
\left(\int_{M}|f|^{4}\right)^{1 / 2} \leqq c\left[\int_{M}|d f|^{2}+|f|^{2}\right]
$$

on $M$. Here $c$ is a constant depending on $M$. If $f$ has compact support on $B_{r}$ then we have the Poincaré-Sobolev inequality

$$
\left(\int_{B_{r}}|f|^{4}\right)^{1 / 2} \leqq c \int_{B_{r}}|d f|^{2} .
$$

These inequalities extend to sections of vector bundles by Kato's inequality $|d| \phi|| \leqq|\nabla \phi|$ for $\phi \in \Gamma^{\infty}(E)$ (we have $|2| \phi|\cdot d| \phi||=|d| \phi||=2|\operatorname{Re}\langle\phi, \nabla \phi\rangle| \leqq 2|\phi| \cdot|\nabla \phi|$ so $|d| \phi|\leqq| \nabla \phi \mid$ on the set where $\phi \neq 0$ or $d|\phi|=0$ and, by continuity, everywhere). Thus, for example, there is a constant $c=c(M, \nabla)$ such that for any $\phi \in L_{1,2}$

$$
\left(\int_{M}|\phi|^{4}\right)^{1 / 2} \leqq c\left[\int_{M}|\nabla \phi|^{2}+|\phi|^{2}\right]
$$

Such inequalities will be used frequently in Sects. 6-8. 
Four dimensional Riemannian geometry provides the background for the field theories we shall be concerned with. We now turn to the dynamical aspects of these theories, introducing the action and the field equations themselves.

\section{Coupled Yang-Mills Equations}

An important guiding principle in geometry is the requirement of naturality. We begin this section by describing the geometrical data necessary for a gauge theory and indicating how naturality determines the theory once this geometric context is fixed.

Let $\pi: P \rightarrow M$ be a principal bundle over an oriented four-dimensional manifold $M$ with compact simple structure group $G$. Let $\varrho: G \rightarrow \operatorname{Aut}(\bar{E})$ be a unitary representation of $G, E=p \times{ }_{e} \bar{E}$ be the associated vector bundle, and let $W$ be any bundle associated to the frame bundle of $M$. The field equations we seek will specify a metric $g$ from the space $\mathscr{M}$ of Riemannian metrics on $M$, a connection $V$ from the space $\mathscr{B}$ of connections on $P$, and a section $\phi$ from the space $\mathscr{E}=\Gamma(E \otimes W)$ of sections of $E$. We shall assume that they are variational equations, that is, they arise as the stationary points on $\mathscr{M} \times \mathscr{C} \times \mathscr{E}$ of an action integral

$$
A(g, \nabla, \phi)=\int_{M} \lambda(g, \omega, \phi)
$$

where the Lagrangian $\lambda$ is a 4-form constructed from $g, \nabla$, and $\phi$.

Now $P$ is a manifold with a certain geometric structure - a free right action of G. An automorphism of $P$ is a map $f: P \rightarrow P$ which preserves this structure: $f\left(x g^{-1}\right)$ $=f(x) g^{-1}$ for all $x \in P$ and $g \in G$. Let $\operatorname{Aut}(P)$ denote the group of all bundle automorphisms $f$ such that the induced map $\pi \circ f: M \rightarrow M$ preserves orientation, and let $\mathrm{Aut}_{0}(P)$ denote the subgroup of automorphisms which induce the identity transformation on $M$. There is an exact sequence

$$
0 \rightarrow \operatorname{Aut}_{0}(P) \rightarrow \operatorname{Aut}(P) \rightarrow \operatorname{Diff}(M),
$$

where $\operatorname{Diff}(M)$ is the group of orientation preserving diffeomorphisms of $M$. The group $\operatorname{Aut}_{0}(P)$ can be identified with the space of sections of the bundle of groups $P \times{ }_{\text {Ad }} G$.

In the terminology of physics, a section $s: M \supset U \rightarrow P$ is called a local choice of gauge, an automorphism $f \in \mathrm{Aut}_{0}(P)$ is a gauge transformation, and the group $\mathscr{G}=\mathrm{Aut}_{0}(P)$ is the gauge group of the bundle.

Note that the Killing form provides an invariant metric $h$ on the adjoint bundle $g$ and hence a hermitian metric on $E$.

We shall require 3 things of the Lagrangian form $\lambda(g, \nabla, \phi)$

(i) regularity - in a local coordinate system and local choice of gauge, $\lambda$ should be given as a universal polynomial in $g, h, \Gamma, \phi,(\operatorname{det} g)^{-1 / 2},(\operatorname{det} h)^{-1 / 2}$ and their derivatives, where $\Gamma$ are the Christoffel symbols of $\nabla$;

(ii) naturality - the map $\lambda: \mathscr{M} \times \mathscr{C} \times \mathscr{E} \rightarrow A^{4}(M)$ should be a natural transformation with respect to the bundle automorphism $f$, i.e.,

$$
\lambda\left((\pi \circ f)^{*} g, f^{*} \nabla, \varrho\left(f^{*}\right) \phi\right)=f^{*} \lambda(g, \nabla, \phi) ;
$$

(iii) conformal invariance - for any function $\sigma$ on $M, \lambda$ satisfies

$$
\lambda\left(e^{2 \sigma} g, \nabla, \phi\right)=\lambda(g, \nabla, \phi) .
$$


Remark. If there is no bundle $P$ present then the naturality property (ii) is Einstein's "principle of general covariance" asserting that the equations of physics are covariant under arbitrary coordinate changes. Naturality with respect to $\operatorname{Aut}_{0}(P)$ means that $\lambda\left(g, f^{*} \nabla, \varrho\left(f^{*}\right) \phi\right)=\lambda(g, \nabla, \phi)$ : this is Weyl's principle of gauge invariance.

We can now use invariant theory to determine the possibilities for $\lambda$. For the case $\lambda=\lambda(g, \nabla)$ if we require naturality under orientation preserving diffeomorphisms of $P$ then SO(4) invariant theory (Stredder [18]) implies that

$$
\lambda=a_{1}|s|^{2}+a_{2}|B|^{2}+a_{3}\left|W^{+}\right|^{2}+a_{4}\left|W^{-}\right|^{2}+a_{5} \Omega \wedge \Omega+a_{6} \Omega \wedge * \Omega,
$$

where $\left\{s, B, W^{ \pm}\right\}$are the components of the Riemannian curvature of $g, \Omega$ is the curvature of $\omega$ and the $a_{i}$ are real numbers. The actions $\int \lambda$ for various values of the $a_{i}$ include the topological invariants $p_{1}(M), \chi(M)$, and $p_{1}(g)$ from (1.11) and (1.12). Of the remaining three independent possibilities two depend on the curvature of $g$; for remarks on these see (Bourguignon and Lawson [6]). Here we will be concerned with the action which depends on the bundle curvature: the Yang-Mills action

$$
A(g, \nabla)=\int_{M} \Omega \wedge * \Omega=\int_{M}|\Omega|^{2} \sqrt{\operatorname{det} g} d x^{1} \wedge \ldots \wedge d x^{4}
$$

This action is evidently regular and $\operatorname{Diff}(M)$ covariant. It is conformally invariant because the $*$ operator on 2 -forms is, or more explicitly

$$
A\left(e^{2 \sigma} g, V\right)=\int_{M} e^{-4 \sigma} g^{i j} g^{k l}\left\langle\Omega_{i k}, \Omega_{j l}\right\rangle\left(\operatorname{det}\left(e^{2 \sigma} g\right)\right)^{1 / 2} d x^{1} \wedge \ldots \wedge d x^{4}=A(g, \omega)
$$

As for gauge invariance, a gauge transformation $g \in \mathscr{G}$ carries $\nabla$ to $g \circ \nabla \circ g^{-1}$ and $\Omega=D \circ \nabla$ to $g \Omega g^{-1}$; the Lagrangian $|\Omega|^{2}$ is then unchanged because the Killing form is invariant.

Since $|\Omega|^{2}=\left|\Omega^{+}\right|^{2}+\left|\Omega^{-}\right|^{2}$, Eq. (1.12) shows that $A(g, \omega) \geqq 8 \pi^{2} k$ with equality if and only if $\Omega^{-} \equiv 0$. Thus self-dual connections are absolute minima of the YangMills action.

It would be interesting to continue with the invariant theory, incorporating the field $\phi$ and finding all Lagrangians satisfying (i), (ii), and (iii). Instead we will simply write down the two action integrals considered by physicists and verify naturality. Following the terminology of physics we will call these the "fermion" and "boson" actions.

Definition. The fermion action is defined on $E$-valued spinors $\phi \in \Gamma(V \otimes E)$ by

$$
A(g, \nabla, \phi)=\int_{M}|\Omega|^{2}+\langle\phi, \mathscr{D} \phi\rangle d v(g),
$$

where $\mathscr{D}$ is the Dirac operator and $\langle$,$\rangle is the inner product on V \otimes E$ and

$$
d v(g)=\sqrt{\operatorname{det} g} d x^{1} \wedge d x^{2} \wedge d x^{3} \wedge d x^{4}
$$

is the volume form of $g$.

Definition. Similarly, the boson action is defined on $E$-valued scalars $\phi \in \Gamma(E)$ by

$$
A(g, \nabla, \phi)=\int_{M}|\Omega|^{2}+\left.|| \nabla \phi\right|^{2}+\frac{s}{6}|\phi|^{2}-P(\phi) d v(g)
$$


where the potential $P: E \rightarrow \mathbb{R}$ is a gauge invariant polynomial on the fiber of degree $\leqq 4$.

Both Lagrangians are regular, Diff $(M)$ covariant, and gauge invariant. The conformal properties of these action integrals will be discussed in the next section.

Remarks. 1. For our purposes the requirement $\operatorname{deg} P \leqq 4$ arises as follows. We wish to vary the action over the Sobolev space $\mathscr{C}_{1,2} \times \mathscr{E}_{1,2}$. By the Sobolev inequality (1.20) any polynomial in $\phi$ of degree $\leqq 4$ is then integrable, while higher degree polynomials need not be.

2. The term $\int\langle\phi, \mathscr{D} \phi\rangle$ in the fermion Lagrangian is not positive definite. Indeed, suppose $\phi=\phi_{+}+\phi_{-} \in \Gamma\left(V_{+} \oplus V_{-}\right)$satisfies $\mathscr{D} \phi=\lambda \phi$ for some eigenvalue $\lambda$. Then $\bar{\phi}=\phi_{+}-\phi_{-}$satisfies $\mathscr{D} \bar{\phi}=-\lambda \bar{\phi}$. Thus the spectrum of $\mathscr{D}$ is symmetric about zero.

Variation of the actions (2.3) and (2.4) yields a complicated system of nonlinear equations for $g, \omega$, and $\phi$; when the Lagrangian $\int s$ of general relatively is included these are complete classical equations of a Yang-Mills particle coupled to gravity (Hawking and Ellis, Sternberg). We shall simplify this system by disregarding the dependence on $g$ (and fixing $h$ ). Because of conformal invariance this amounts to fixing the conformal structure of $M$.

With this agreed, the next step is to calculate the Euler-Lagrange equations of the fermion and boson actions.

\section{The Field Equations}

For a spinor field $\phi \in \Gamma(V \otimes E)$ the first variation of the action (2.3) is computed as follows. Choose a 1-parameter family of connections $\nabla_{s}=\nabla_{0}+s \eta+\ldots$, $\eta \in \Gamma\left(A^{1} \otimes g\right)$ and a 1 -parameter family of spinors $\phi_{t}=\phi_{0}+\mathrm{t} \psi+\ldots, \psi \in \Gamma(\mathrm{V} \otimes \mathrm{E})$. The curvature and total covariant derivative on $V \otimes E$ are [cf. (1.3) and (1.7)]

$$
\left\{\begin{array}{l}
\Omega_{s}=\Omega_{0}+s D_{0} \eta+\frac{s}{2}[\eta, \eta] \\
\nabla_{s}=\nabla_{0}+s \varrho(\eta)
\end{array}\right.
$$

Hence

$$
\begin{aligned}
A\left(\nabla_{s}, \phi_{t}\right)= & \int_{M}\left|\Omega_{0}\right|^{2}+2 s\left\langle\Omega_{0}, D_{0} \eta\right\rangle+t\langle\psi, \mathscr{D} \phi\rangle+t\langle\phi, \mathscr{D} \phi\rangle \\
& +s\left\langle\phi, \sum e^{i} \cdot \varrho\left(\eta_{i}\right) \phi\right\rangle+\ldots,
\end{aligned}
$$

where $\left\{e^{i}\right\}$ is a local orthonormal frame and we have retained only the terms linear in $s$ and $t$. The first variation equations are

$$
\begin{cases}0=\int_{M} 2\left\langle D^{*} \Omega, \eta\right\rangle+\left\langle\phi, \sum e^{i} \cdot \varrho\left(\eta_{i}\right) \phi\right\rangle & \forall \eta \in \Gamma^{\infty}\left(A^{1} \otimes g\right) \\ 0=\int_{M}\langle\psi, \mathscr{D} \phi\rangle+\langle\phi, \mathscr{D} \psi\rangle & \forall \psi \in \Gamma^{\infty}(V \otimes E)\end{cases}
$$

or (noting that $\mathscr{O}$ is self-adjoint)

$$
\left\{\begin{array}{l}
D^{*} \Omega=J(\phi)=-\frac{1}{2} \sum\left\langle\phi, e^{i} \cdot \varrho\left(\sigma^{\alpha}\right) \phi\right\rangle \sigma_{\alpha} \otimes e_{i} \\
\mathscr{D} \phi=0
\end{array}\right.
$$


where $\left\{\sigma_{\alpha}\right\}$ is a local orthogonal basis of sections of $g,\left\{\sigma^{\alpha}\right\}$ the dual basis in $\Gamma\left(g^{*}\right)$. Here $J(\phi)$ is the current due to the field $\phi$; it is real-valued since $\left\langle\phi, e^{i} \cdot \varrho\left(\eta_{i}\right) \phi\right\rangle$ $=-\left\langle e^{i} \cdot \phi, \varrho\left(\eta_{i}\right) \phi\right\rangle=\left\langle e^{i} \cdot \varrho\left(\eta_{i}\right) \phi, \phi\right\rangle$ and is thus an element of $\left(A^{1}\right)^{*}$. It should be interpreted as a 1 -form on the space of connections $\mathscr{C}$.

The calculation of the first variation of the boson action (2.4) is similar.

More generally we shall replace the second field equation by the corresponding eigenvalue equation. In the fermion case this is $\mathscr{D} \phi=m \phi$, where $m$ is a real constant ( $\mathscr{D}$ is self-adjoint). In the boson case there are two possibilities: the eigenvalue equation $\nabla^{*} \mid \nabla \phi-\frac{s}{6} \phi=m^{2} \phi$ or the equation with potential $\left|\nabla^{*}\right| \nabla \phi$ $-\frac{s}{6} \phi=P^{\prime}(\phi)$; the latter equation is particularly important when $P$ is the Higgs potential $P(\phi)=|\phi|^{4}-|\phi|^{2}$. We shall deal with both possibilities simultaneously by taking $P^{\prime}$ of the form $P^{\prime}=a|\phi|^{2} \phi+m^{2} \phi$, where $a$ and $m^{2}$ are real with $a=0$ and $m^{2} \leqq 0$ allowable.

Thus the equations which we shall study are the coupled fermion equations

$$
\left\{\begin{array}{l}
D^{*} \Omega=J=-\frac{1}{2} \sum\left\langle\phi, e^{i} \cdot \varrho\left(\sigma^{\alpha}\right) \phi\right\rangle \quad \sigma_{x} \otimes e_{i} \\
\mathscr{D} \phi=m \phi,
\end{array}\right.
$$

and the coupled boson equations

$$
\left\{\begin{array}{l}
D^{*} \Omega=J=-\operatorname{Re} \sum\left\langle\mid \nabla_{i} \phi, \varrho\left(\sigma^{\alpha}\right) \phi\right\rangle \quad \sigma_{x} \otimes e_{i} \\
\left|\nabla^{*}\right| \nabla \phi=\frac{s}{6} \phi+a|\phi|^{2} \phi+m^{2} \phi .
\end{array}\right.
$$

In physics, $\Omega$ is a gauge field, $\omega$ its potential, $\phi$ is the field of a particle interacting with $\Omega$ and $m$ is the mass of the particle. For example, when the structure group is $S^{1}(2.5)$ describes an electron interacting with an electromagnetic field.

When $\phi \equiv 0$ the fermion and boson actions reduce to the Yang-Mills action and the field equations become the Yang-Mills equation $D^{*} \Omega=0$. Self-dual connections satisfy this equation because they are absolute minima of the action. This also follows from the Bianchi identity: if $\Omega$ is self-dual $\Omega=* \Omega$ and $D^{*} \Omega$ $=* D \Omega=0$.

The current $J \in A^{1 *}$ which appears in the field equations is a $D$-coclosed form. This is a consequence of a gauge invariance. In fact, evaluating $D^{*} J$ on $X \in \Gamma(g)$ gives $D^{*} J \cdot X=J(\mid V X)$. But $\nabla X$ is an infinitesimal gauge transformation [see (4.1) below] and $J$ is constant on gauge orbits; hence $\int J(\mid \nabla X)=0$ and $D^{*} J \equiv 0$.

The equation $D^{*} J=0$ also follows from direct calculation. For example, in the fermion case $* J=\frac{1}{2}\left\langle\phi, e^{i} \cdot \varrho\left(\sigma^{\alpha}\right) \phi\right\rangle \sigma^{\alpha} \otimes * e^{i}$ and, in an orthonormal frame $\left\{e^{i}\right\}$ with $\nabla_{i} e^{i}=\nabla_{i} \sigma_{\alpha}=0$ at a point $x \in M$, the field equations give

$$
\begin{aligned}
D * J & =-\operatorname{Re}\left\langle\nabla_{i} \phi, e^{i} \cdot \varrho\left(\sigma^{\alpha}\right) \phi\right\rangle \sigma_{\alpha}=\operatorname{Re}\left\langle e^{i} \cdot \mid \nabla_{i} \phi, \varrho\left(\sigma^{\alpha}\right) \phi\right\rangle \sigma_{\alpha} \\
& =\operatorname{Re}\left\langle m \phi, \varrho\left(\sigma^{\alpha}\right) \phi\right\rangle \sigma_{\alpha}=0
\end{aligned}
$$

since $\left\langle\phi, \varrho\left(\sigma^{\alpha}\right) \phi\right\rangle$ is pure imaginary. Alternatively, we can use the first field equation: for $\Omega=\Omega_{\alpha} \otimes \sigma^{\alpha} \in \Lambda^{2} \otimes g$ we have

$$
D * J=D D * \Omega=[\Omega, * \Omega]=* \sum_{\alpha, \beta}\left\langle\Omega_{\alpha}, \Omega_{\beta}\right\rangle \cdot\left[\sigma^{\alpha}, \sigma^{\beta}\right]=0
$$


When the structure group $G$ is abelian the equation $D^{*} J=0$ becomes $d^{*} J=0$. This is the equation of (infinitesimal) conservation of charge in electromagnetism.

\section{Gap Theorems}

The field equations (2.5) and (2.6) are simplest when $a=m=0$. Then $\phi$ satisfies either $\mathscr{D} \phi=0$ ( $\phi$ on $E$-valued spinor) or $\nabla^{*} \nabla \phi=\frac{s}{6} \phi$ ( $\phi$ an $E$-valued scalar).

Proposition 2.1. Let $E$ be a vector bundle over a manifold $M$ and let $(\phi, \Omega)$ be a solution to the coupled boson equations $D^{*} \Omega=J, \nabla^{*} \mid \nabla \phi=\frac{s}{6} \phi$. If $M$ is compact with positive scalar curvature, or if $M=\mathbb{R}^{4}$ and $\phi$ vanishes at infinity, then $\phi=0$ and $\Omega$ is a Yang-Mills field.

Proof. When $M$ is compact and $s>0$ integration by parts gives

$$
0=\left.\int_{M}|| \nabla \phi\right|^{2}+\frac{S}{6}|\phi|^{2}
$$

so $\phi \equiv 0$ and $J \equiv 0$. On $\mathbb{R}^{4}$, we can convert the equation $\left|\nabla^{*}\right| \nabla \phi=0$ into a differential inequality for $|\phi|$ by

$$
\begin{aligned}
& d^{*} d|\phi|^{2}=2 d^{*}\langle\phi, \mid \nabla \phi\rangle=-\left.2|| \nabla \phi\right|^{2}+2\left\langle\phi,\left|\nabla^{*}\right| \nabla \phi\right\rangle=-\left.2|| \nabla \phi\right|^{2}, \\
& d^{*} d|\phi|^{2}=2 d^{*}(|d| \phi)=-2|d| \phi||^{2}+2|\phi| \cdot d^{*} d|\phi| .
\end{aligned}
$$

These give $|\phi| d^{*} d|\phi|=|d| \phi \|^{2}-\left.|| \nabla \phi\right|^{2} \leqq 0$, or $\Delta|\phi| \geqq 0$. If $|\phi|$ vanishes at infinity the maximum principal (Morrey, p. 61) implies that $\phi=0$; hence the current $J=\langle\nabla \phi, \varrho(\cdot) \phi\rangle$ vanishes, and $\Omega$ is a Yang-Mills field.

For the pure Yang-Mills equations and for the coupled fermion equations, we have the following $L_{2}$ "gap theorem" (part (a) is essentially due to Bourguignon and Lawson [6]).

Proposition 2.2. Let $M$ be a compact Riemannian 4-manifold with $\frac{s}{3}-\left|W^{-}\right| \geqq \varepsilon>0$.

Then there is a constant $c_{0}$ such that

(a) Any Yang-Mills $\Omega$ with $\left\|\Omega^{-}\right\|_{0,2}<c_{0}$ is self-dual.

(b) Any solution $(\Omega, \phi)$ to the massless coupled fermion equations (2.5) with $\left\|\Omega^{-}\right\|_{0,2}<c_{0}$ satisfies $\Omega^{-} \equiv J \equiv \phi^{-} \equiv 0$.

Proof. (a) From $0=D^{*} \Omega=D \Omega$ we obtain $0=D \Omega^{+} \pm D \Omega^{-}, 0=D \Omega^{-}=D^{*} \Omega^{-}$and hence $\square \Omega^{-}=0$. Integrating $\left\langle\Omega^{-}, \square \Omega^{-}\right\rangle$over $M$, using (1.19), integrating by parts and applying Kato's inequality gives

$$
0 \geqq\left.\int_{M}|d| \Omega^{-}\right|^{2}+\varepsilon\left|\Omega^{-}\right|^{2}-\left|\Omega^{-}\right|^{3} .
$$

By the Hölder and Sobolev inequalities the last term is bounded by $c\left\|\Omega^{-}\right\|_{0,2}\left(\left\|d \Omega^{-} \mid\right\|_{0,2}^{2}+\left\|\Omega^{-}\right\|_{0,2}^{2}\right)$, which is dominated by the first two terms whenever $\left\|\Omega^{-}\right\|_{0,2}$ is sufficiently small. This is a contradiction unless $\Omega^{-} \equiv 0$. 
(b) If $\phi=\left(\phi^{+}, \phi^{-}\right) \in V_{+} \oplus V_{-}$satisfies $\mathscr{D} \phi=0$, then $\left\langle\phi, \mathscr{D}^{2} \phi^{-}\right\rangle=0$. Using (1.14) on $V^{-}$and repeating the above argument shows that $\phi^{-} \equiv 0$ whenever $\left\|\Omega^{-}\right\|_{0,2}$ is sufficiently small. But then

$$
-2 D^{*} \Omega=-2 J=\left\langle\phi^{-}, e^{i} \cdot \varrho() \phi^{+}\right\rangle+\left\langle\phi^{+}, e^{i} \cdot \varrho() \phi^{\prime}\right\rangle \equiv 0
$$

and $\Omega^{-} \equiv 0$ by part (a).

This argument and the fact that $\|f\|_{0,4} \leqq c\|d f\|_{0,2}$ for $f \in L_{1,2}\left(\mathbb{R}^{4}\right)$ also shows that Proposition 2.2 holds for $M=\mathbb{R}^{4}$.

\section{Conformal Invariance and Rescaling}

From physics we know that the Lagrangian and the energy must be conformally ("scale") invariant. The Lagrangians introduced in Sect. 2 do not, a priori, have this property. We will now show how to arrange conformal invariance by further specifying the geometric properties of the field $\phi$.

Let $M^{n}$ be an oriented manifold and $F$ the GL(n) frame bundle of its cotangent bundle $\tau M$. A choice of conformal structure on $M$ reduces $F$ to a principal bundle $F_{\varepsilon}$ whose structure group is the conformal group $\mathrm{CO}(n)=\mathbb{R}^{+} \times \mathrm{SO}(n)$. The representations of $\mathrm{CO}(n)$ are specified by pairs $(w, \varrho)$ where $w \in \mathbb{R}^{+}$and $\varrho: \mathrm{SO}(n) \rightarrow \mathrm{GL}(W)$ is a representation of $\mathrm{SO}(n)$; the representation $(w, \varrho)$ takes the matrix $B=\lambda^{1 / n} A$, where $\lambda=|\operatorname{det} B|$ and $A \in \mathrm{O}(n)$, to $\lambda^{w} \varrho(A)$. The number $w$ is the conformal weight of the representation and of the corresponding bundle $F_{c} \times{ }_{(w, e)} W$.

In this context there are two vector bundles of primary importance: a trivial real line bundle $L=F_{c} \times{ }_{(1,1)} \mathbb{R}$ with weight 1 and an orthogonal bundle $T=F_{c} \times{ }_{(0, \text { Id })} \mathbb{R}^{n}$ with weight 0 . A choice of a metric within the conformal class, trivializes $L$ and $\tau^{*} M$ then becomes an orthogonal bundle under the conformal isomorphism $\tau^{*} M=T \otimes L$.

If $M^{4}$ has a spin structure, $F_{c}$ lifts to a conformal spin bundle $\tilde{F}_{c}$ with structure group $\widetilde{C O}(n)=\mathbb{R} \times \operatorname{Spin}(n)$. Spinors of weight $w$ are then sections of

$$
V_{ \pm}^{w}=\tilde{F}_{c} \times_{\left(w, V_{ \pm}\right)} \mathbb{C}^{2}=V_{ \pm}^{0} \otimes L^{w}
$$

A metric $g$ on $M$ provides a trivialization of $L$ and a Riemannian connection on $\tau^{*} M$ and $T$. This connection on $T$ induces one on $V^{0} \simeq V^{w}$ and allows us to define a Dirac operator $\mathscr{D}: \Gamma\left(V^{w}\right) \rightarrow \Gamma\left(V^{w} \otimes L\right)$ for each conformal weight $w$. More generally, if $E$ is a vector bundle associated to a principal bundle $P$ over $M$ then a connection on $P$ gives Dirac operators $\mathscr{D}: \Gamma\left(V^{w} \otimes E\right) \rightarrow \Gamma\left(V^{w+1} \otimes E\right)$.

Replacing $g$ by a conformally equivalent metric $g^{\prime}=s^{2 \sigma} g$ changes the Riemannian connection on $\tau^{*} M$ and the trivialization of $L$, and transforms the Dirac operator $\mathscr{D}$ on $\Gamma\left(V^{w}\right)$ to $\mathscr{D}^{\prime}$ given by (cf. Hitchen [9])

$$
\mathscr{D}^{\prime} \phi=\mathscr{D} \phi-\frac{3}{2} d \sigma \cdot \phi+w d \sigma \cdot \phi .
$$

In particular, the Dirac operator on spinors of weight $3 / 2$ is independent of the metric within the conformal class (see also Fegan [7]). 
This conclusion also follows by examining the spinor Lagrangian

$$
\int_{M}\langle\phi, \mathscr{D} \phi\rangle \sqrt{\operatorname{det} g} d x^{1} \wedge d x^{2} \wedge d x^{3} \wedge d x^{4} \quad \phi \in \Gamma\left(V^{w} \otimes E\right) .
$$

This integral is invariant under constant conformal changes $g \mapsto e^{2 \sigma} g, \sigma \in \mathbb{R}$, when the integrand has weight 4 . But $\phi \in \Gamma\left(V^{w} \otimes E\right)$ has weight $w$ (assuming $E$ is weightless) and $\mathscr{Q} \phi \in \Gamma\left(V^{w} \otimes E \otimes L\right)$ has weight $w+1$. Thus the Lagrangian, and the corresponding field equations, are scale invariant on spinors of weight $w$, when $2 w+1=4$, i.e., $w=\frac{3}{2}$. Invariance under arbitrary conformal changes follows from (3.1).

The equation $\mathscr{D} \phi=\mathrm{m} \phi$ is conformally invariant if we interpret $m$ as a section of $L: \mathscr{D} \phi=\phi \otimes m \in V^{3 / 2} \otimes L$. Then both $\mathscr{D} \phi$ and $m \phi$ have weight $\frac{5}{2}$.

The boson equations for scalar fields can likewise be made conformally invariant. In this case the Lagrangian is basically [cf. (2.4)]

$$
\int_{M}\langle|\nabla \phi,| \nabla \phi\rangle \sqrt{\operatorname{det} g} d x^{1} \wedge d x^{2} \wedge d x^{3} \wedge d x^{4} \quad \phi \in \Gamma(E \otimes L) .
$$

If $\phi$ has weight $w, \nabla \phi$ has weight $w+1$ and this Lagrangian is invariant under constant scale changes when $2 w+2=4$ and $w=1$. Thus we take $\phi$ to be a section of $E \otimes L$.

While this Lagrangian (3.3) and the corresponding Laplacian are not invariant under arbitrary scale changes, there does exist a modified Laplacian which is conformally invariant. To find it we return to the viewpoint taken in Sect. 2 and seek a second order weight 2 operator $A$ on $\Gamma(E \otimes L)$ constructed naturally from the metric on $M$, the connection on $E$, and their derivatives. By invariant theory (Atiyah, Bott, and Patodi and Stredder) $A=\left|F^{*}\right| \nabla+p\left(R, \Omega^{\bar{E}}\right)$. where $\left|F^{*}\right| V$ is the trace Laplacian on $E \otimes L$ and $p\left(R, \Omega^{E}\right)$ is a polynomial in the curvature tensors $R$ of $M$ and $\Omega^{E}$ of $E$. But $p\left(R, \Omega^{E}\right) \in \operatorname{End}(E \otimes L)$ must have weight 2 ; the invariant theory then shows that $A=\left|\nabla^{*}\right| \nabla+a \cdot$ s for some constant $a$. A direct calculation (see Hitchen [10]) shows that this is indeed conformally invariant when $a=1 / 6$, that is, solutions of $\left(\left|\nabla^{*}\right| \nabla+\frac{s}{6}\right) \phi=0$ remain solutions after a conformal change of metric.

In the corresponding field equations with mass, $\left|\nabla^{*}\right| \nabla \phi=\frac{s}{6} \phi+m^{2} \phi, m$ is again interpreted as a section of $L$. Likewise, in the potential $P(\phi)=a|\phi|^{4}+m^{2}|\phi|^{2}$, $a$ has conformal weight zero and $m \in \Gamma(L)$.

The above argument also shows that the Lagrangian

$$
\left.\int_{M}|| \nabla \phi\right|^{2}+\frac{s}{6}|\phi|^{2} d v_{g}
$$

is scale invariant. Together with the conformal invariance of (2.2) and (3.2), this completes the verification that the fermion and boson Lagrangians (2.5) and (2.6) meet the requirements of Sect. 2: they are regular, natural, and conformally invariant.

We conclude this section by describing several useful devices, related to conformal invariance, which will be important later. 
The first of these is the total field $F$. To define it, fix a metric $g_{0}$ on $M$ and let $\tau \in \Gamma\left(L^{1 / 2}\right)$ be the function of weight $\frac{1}{2}$ with $\tau \equiv 1$ in the trivialization of $L^{1 / 2}$ determined by $g_{0}$.

Definition 3.1. The total field $F$ is the section of a weight 2 bundle $\mathscr{F}$ defined as follows.

(i) When $(\phi, \Omega)$ is a solution of the fermion equations $F=\Omega+\tau^{-1} e^{k} \otimes \nabla_{k} \phi+\tau \phi\left(\left\{e^{k}\right\}\right.$ is an orthonormal coframe $)$ is a section of

$$
\mathscr{F}=\left(A^{2} T \otimes g \otimes L^{2}\right) \oplus\left(T \otimes L^{1 / 2} \otimes V^{3 / 2} \otimes E\right) \oplus\left(L^{1 / 2} \otimes V^{3 / 2} \otimes E\right) .
$$

(ii) When $(\phi, \Omega)$ is a solution of the boson equations (2.6), $F=\Omega+e^{k} \otimes \mid \nabla_{k} \phi+\tau^{2} \phi$ is a section of

$$
\mathscr{F}=\left(\Lambda^{2} T \otimes \mathscr{g} \otimes L^{2}\right) \oplus(T \otimes L \otimes E \otimes L) \oplus(L \otimes E \otimes L) .
$$

When $\mathscr{F}$ is given the direct sum metric, $|F|^{2} d v_{g}$ is a conformally invariant energy density for the field $(\phi, \Omega)$. Fix a point $p \in M$ and let $B(\tau, r)$ be the ball of radius $r$ in the metric $g=\tau^{-4} g_{0}$. The local energy integral of $(\phi, \Omega)$ around $p$ is

$$
\begin{aligned}
& E(\tau, r)=\int_{B(\tau, r)}|F|^{2}=\int_{B(\tau, r)}|\Omega|^{2}+\left.\left|\tau^{-1}\right| \nabla \phi\right|^{2}+|\tau \phi|^{2}, \\
& E(\tau, r)=\int_{B(\tau, r)}|F|^{2}=\int_{B(\tau, r)}|\Omega|^{2}+\left.|| \nabla \phi\right|^{2}+\left|\tau^{2} \phi\right|^{2}
\end{aligned}
$$

in the fermion and boson cases respectively. $E(\tau, r)$ thus depends on both the scale $\tau$ and the radius $r$; we will abbreviate it to $E(r)$ when the scale has been fixed. The conformal invariance of $|F|^{2} \cdot d v_{g}$ implies that $E(\tau, r)=E\left(1, \tau^{2} r\right)$.

Suppose that $|F|^{2}$ is integrable on some neighborhood $U$ of $p \in M$. Then, given $\varepsilon>0$, we can find an $R$ such that $B(1,2 R) \subset U$ and $E(1,2 R)<\varepsilon$. Now change the metric to $g=R^{-2} g_{0}$. Under this rescaling solutions of conformally invariant equations remain solutions, while $B(1,2 R)$ becomes $B(2)$ and the energy in the new metric satisfies $E(2)=E\left(R^{1 / 2}, 2\right)=E(1,2 R)<\varepsilon$. Thus we have the

\section{Rescaling Principle}

Given $\varepsilon>0$ and a solution $(\phi, \Omega)$ to conformally invariant field equations defined in a neighborhood of $p \in M$ with locally finite energy, we may assume - by a constant conformal change of metric - that $(\phi, \Omega)$ is a solution on $B(2)$ to the field equations (with rescaled $m$ and $s$ ) and that $E(2)<\varepsilon$.

\section{A Slice Theorem and the Orbit Space}

In this section we prove a local slice theorem for the action of the gauge group on $\mathscr{C} \times \mathscr{E}$. This extends similar theorems which appear in Atiyah et al. [4] and Uhlenbeck [20].

The center of the gauge group - the sections of the trivial bundle $P \times{ }_{\mathrm{Ad}} Z$, where $Z$ is the center of $G$-acts trivially on the space of connections. Thus we will deal with the group $\mathscr{G}_{e}=\Gamma\left(P \times{ }_{\mathrm{Ad}} G / Z\right)$ of effective gauge transformations. To topologize $\mathscr{G}_{e}$, note that the adjoint representation is a faithful representation Ad: $G / Z \rightarrow \operatorname{End}(g)$ and hence defines an embedding $\mathscr{G}_{e} \rightarrow \Gamma\left(P \times{ }_{\text {Ad }} \operatorname{End}(g)\right)$. This 
latter bundle inherits a connection from any given $\nabla \in \mathscr{C}$ and the Sobolev completion $\mathscr{G}_{k, p}$ of the effective gauge group - with norm determined by $\nabla$ appears as a closed subspace of $L_{k, p}\left(P \times{ }_{\mathrm{Ad}} \operatorname{End}(g)\right)$ ). For $p k>4, \mathscr{G}_{k, p}$ is a smooth Lie group, any $g \in \mathscr{G}_{k, p}$ is continuous, and the map $X \mapsto \exp (X)$ induces a smooth (nonlinear) map from a neighborhood of 0 in $A_{k, p}^{0}$ into $\mathscr{G}_{k, p}$. This is a standard result. Mitter and Viallet [12], for example, give a proof for $p=2$, and their proof is valid for $p k>4$ because multiplication induces a continuous map $L_{k, p} \times L_{k, p} \rightarrow L_{k, p}$ (Palais, Sect. 9). The effective gauge group $\mathscr{G}_{e}$ also acts on $\mathscr{E}=\Gamma\left(P \times{ }_{e} \bar{E}\right)$ if $Z \subset$ ker $\varrho$; this occurs if the highest weight of $\varrho$ lies on the root lattice of $g^{*}$. In this section we will assume that $\varrho$ is such a representation.

We first determine the equation for the orbit of $\mathscr{G}_{e}$ in a neighborhood of a $C^{\infty}$ field $(\nabla, \phi) \in \mathscr{C} \times \mathscr{E}$. This connection $\nabla$ provides an identification $\mathscr{C}=A^{1}$ by $\nabla+\eta \leftrightarrow \eta$. Choose a gauge transformation $g$ close to the identity and write $g=\exp (X), X \in A^{0}$. Then $g$ transforms the connection $\nabla+\eta$ to

or

$$
g \cdot(\nabla+\eta)=\nabla+g \nabla g^{-1}+(\mathrm{Ad} g) \eta
$$

$$
\exp (X)(\nabla+\eta)-\nabla=\exp (X) V \exp (-X)+\operatorname{Ad}[\exp (X)] \eta .
$$

This expression is non-linear in $X$; we can write it as its linearization plus a remainder:

$$
\exp (X)(\nabla+\eta)-\nabla=\eta+\nabla X+R(X, \eta) .
$$

$R(X, \eta)$ is a power series of brackets of $X, \nabla X$, and $\eta$, and $R(t X, t \eta)=O\left(t^{2}\right)$.

Now suppose that there is a field $\phi \in \mathscr{E}=\Gamma(W \otimes E)$ present ( $W$ is any bundle associated to the frame bundle of $M$ ). Write nearby fields as $\phi+\psi$ where $\psi \in \mathscr{E}$ is small. Then $g=\exp (X)$ transforms $\phi+\psi$ to $\varrho(g)(\phi+\psi)$, or

$$
\exp (X)(\phi+\psi)-\phi=\psi+\varrho(X)(\phi+\psi)+S(X, \phi+\psi),
$$

where $S(t X, \phi+\psi)=O\left(t^{2}\right)$.

It follows from these transformation formulae that the action of the gauge group induces a smooth map

$$
\mathscr{G}_{k+1, p} \times(\mathscr{C} \times \mathscr{E})_{k, p} \rightarrow(\mathscr{C} \times \mathscr{E})_{k, p}
$$

for $(k+1) p>4$ (cf. Uhlenbeck [20]).

It is also apparent from Eq. (4.1) and (4.2) that the tangent space to the orbit of the gauge group through $(\nabla, \phi) \in \mathscr{C} \in \mathscr{E}$ is the image of

$$
K: A^{0} \rightarrow A^{1} \times \mathscr{E} \quad \text { by } X \mapsto(\nabla X, \varrho(X) \phi) .
$$

The $L^{2}$ orthogonal complement of the image - which is the kernel of the adjoint operator $K^{*}$ - provides a natural slice for the gauge orbit. This adjoint is $K^{*}:(\eta, \psi) \mapsto \nabla^{*} \eta+\langle\psi, \varrho() \phi\rangle$, where this last term specifies an element of $A^{0}=\left(A^{0}\right)^{*}$ via the Killing metric and evaluation. More generally, for each $0 \leqq \lambda \in \mathbb{R}$ we can consider the subspace $\operatorname{ker} K_{\lambda}^{*}$ where

$$
K_{\lambda}^{*}:(\eta, \psi) \mapsto \nabla^{*} \eta+\lambda\langle\psi, \varrho() \phi\rangle .
$$

From (4.1), (4.2), and (4.3) we see that $0=K_{\lambda}^{*}[g \cdot(\nabla+\eta)-\nabla, g \cdot \phi-\phi]$ if and only if

$$
\begin{aligned}
0= & \nabla^{*} \nabla X+\nabla^{*} \eta+\nabla^{*} R(X, \eta)+\lambda\langle\psi, \varrho() \phi\rangle+\lambda\langle\varrho(X)(\phi+\psi), \varrho() \phi\rangle \\
& +\lambda\langle S(X, \phi+\psi), \varrho() \phi\rangle .
\end{aligned}
$$


This is an elliptic equation for $X$, and if $X$ is a solution then $g=\exp (X)$ is a gauge transformation which carries $(\nabla+\eta, \phi+\psi)$ onto the slice $\operatorname{ker} K_{\lambda}^{*}$ through $(\nabla, \phi)$.

The slice theorem will apply at the regular points of $\mathscr{C} \times \mathscr{E}$ - the open dense set of points $(\nabla, \phi)$ where $\operatorname{ker} K_{\lambda}=\left\{X \in A_{k+2, p}^{0} / \nabla X=0\right.$ and $\left.\varrho(X) \phi=0\right\}$ is zero (i.e. where the action of the gauge algebra is free).

Theorem 4.1. Let $M$ be a compact Riemannian 4-manifold, possibly with boundary. Fix a regular field $(\nabla, \phi) \in(\mathscr{C} \times \mathscr{E})_{k+1, p}$ with $k \geqq 0,2<p<4$. Then there is a constant $c_{0}$ such that for every field $(\eta, \psi) \in\left(A^{1} \times \mathscr{E}\right)_{k+1, p}$ with $\|(\eta, \psi)\|_{k+1, p}<c_{0}$ there is a gauge transformation $g \in \mathscr{C}_{\boldsymbol{k}+2, p}$, unique in a neighborhood of the identity, with

$$
K_{\lambda}^{*}(g \cdot(\nabla+\eta)-\nabla, \quad g \cdot(\phi+\psi)-\phi)=0
$$

weakly. If $\nabla, \phi, \eta$, and $\psi$ are $C^{\infty}$ then $g$ is $C^{\infty}$.

Proof. The proof is an application of the Banach space implicit function theorem. Let $T \subset A_{k+2, p}^{0}$ be the subspace of $X \in A_{k+2, p}^{0}$ which vanish on $\partial M\left(T=A_{k+2, p}^{0}\right.$ if $\partial M=\phi)$. Let $F$ be the map

$$
T \times\left(A^{1} \times \mathscr{E}\right)_{k+1, p} \rightarrow A_{k, p}^{0}
$$

defined by $F(X,(\eta, \psi))=$ right-hand side of (4.4). Since multiplication is a continuous map

$$
L_{k+1, p} \times L_{k+1, p} \rightarrow L_{k, p} \text { and } L_{k+2, p} \times L_{k+1, p} \rightarrow L_{k+1, p}
$$

(Palais, Sect. 9) we have

$$
\begin{aligned}
\|F(X,(\eta, \psi))\|_{k, p} \leqq & c\left(\|X\|_{k+2, p}+\|\eta\|_{k+1, p}+\|R(X, \eta)\|_{k+1, p}\right. \\
& +\lambda\|\psi\|_{k+1, p}\|\phi\|_{k+1, p}+\lambda\|X\|_{k+2, p} \cdot\|\phi\|_{k+1, p} \cdot\|\phi+\psi\|_{k+1, p} \\
& \left.+\lambda\|\phi\|_{k+1, p} \cdot\|S(X, \phi+\psi)\|_{k+1, p}\right) .
\end{aligned}
$$

When the norms of $X, \eta$, and $\psi$ are small, the continuity of exp: $A_{k+2, p}^{0} \rightarrow \mathscr{G}_{k+2, p}$ implies that

$$
\begin{aligned}
\|S(X, \phi+\psi)\|_{k+1, p} & =\|\exp (X)(\phi+\psi)-(\phi+\psi)-\varrho(X)(\phi+\psi)\|_{k+1, p} \\
& \leqq c\|\phi+\psi\|_{k+1, p} \cdot\|X\|_{k+2, p},
\end{aligned}
$$

and similarly $\|R(X, \eta)\|_{k+1, p} \leqq c\left(\|X\|_{k+2, p}+\|\eta\|_{k+1, p}\right)$. Thus there are neighborhoods $U$ of zero in $T$ and $V$ of zero in $\left(A^{1} \times \mathscr{E}\right)_{k+1, p}$ such that $F$ is a continuous map $F: U \times V \rightarrow A_{k, p}^{0}$. Similar estimates show that $F$ is $C^{1}$.

The differential of $F$ at zero is $D_{1} F(0,0) X=K^{*} K X=\nabla^{*} \nabla X+\lambda(\varrho(X) \phi, \varrho() \phi\rangle$. For $k \geqq 1$ multiplication is a continuous map $L_{k+1, p} \times L_{k+1, p} \times L_{k, p} \rightarrow L_{k, p}$ (see [14]) so

$$
\|\| K^{*} K X\left\|_{k, p}-\right\| \nabla^{*} \nabla X\left\|_{k, p} \mid \leqq c_{1}\right\| \phi\left\|_{k+1, p}^{2}\right\| X \|_{k, p},
$$

while for $k=0$ this difference is bounded by $\|\phi\|_{1, p}^{2} \cdot\|X\|_{\infty} \leqq c_{2}\|X\|_{2, p}$; in both cases $\left\|K^{*} K X\right\|_{k, p} \leqq c_{3}\|X\|_{k+2, p}$. The elements of $T$ satisfy the Poincaré inequality $\|X\|_{0,2} \leqq c_{4}\|K X\|_{0,2}$. Integrating by parts,

$$
\|X\|_{0,2} \leqq c_{4}\|K X\|_{0,2} \leqq c_{4}\left(\int\left\langle X, K^{*} K X\right\rangle\right)^{1 / 2} \leqq \varepsilon c_{4}\|X\|_{0,2}+c_{4} \varepsilon^{-1}\left\|K^{*} K X\right\|_{0,2}
$$


for any $\varepsilon>0$; choosing $\varepsilon$ sufficiently small and subtracting yields $\|X\|_{0,2} \leqq c_{5}\left\|K^{*} K X\right\|_{0, p^{*}}$ Also, using Kato's inequality as in the proof of Proposition 2.1,

$$
|X| d^{*} d|X| \leqq\left\langle X, \nabla^{*} \nabla X\right\rangle=\left\langle X, K^{*} K X\right\rangle-|\varrho(X) \phi|^{2} \leqq|X|\left|K^{*} K X\right|,
$$

so $d^{*} d|X| \leqq\left|K^{*} K X\right|$ pointwise and by standard theory (Morrey [13, Corollary 5.3.1])

$$
\|X\|_{\infty} \leqq c_{6}\left(\left\|K^{*} K X\right\|_{0,2}+\|X\|_{0,2}\right) \leqq c_{7}\left\|K^{*} K X\right\|_{0, p}
$$

Hence

$$
\|X\|_{2, p} \leqq\left\|V^{*} \nabla X\right\|_{0, p}+c_{8}\|X\|_{0,2} \leqq\left(1+c_{2} c_{7}+c_{5} c_{8}\right)\left\|K^{*} K X\right\|_{0, p}
$$

For $k \geqq 1$ interpolation gives

$$
\|X\|_{k+2, p} \leqq\left\|K^{*} K X\right\|_{k, p}+c_{1}\|\phi\|_{k+2, p}^{2}\left(\varepsilon\|X\|_{k+2, p}+c(\varepsilon)\|X\|_{0,2}\right)+c_{9}\|X\|_{0,2}
$$

and - again choosing $\varepsilon$ small $-\|X\|_{k+2, p} \leqq c_{10}\left\|K^{*} K X\right\|_{k, p}$. Thus

$$
c_{3}^{-1}\left\|K^{*} K X\right\|_{k, p} \leqq\|X\|_{k+2, p} \leqq c\left\|K^{*} K X\right\|_{k, p}
$$

for all $k$, so $D_{1} f$ is an isomorphism.

By the implicit function theorem there is a map $(\eta, \psi) \mapsto X(\eta, \psi)$ uniquely defined on a possibly smaller neighborhood of the origin, such that $F(X(\eta, \psi),(\eta, \psi))=0$. $X(\eta, \psi)$ then satisfies (4.4) and the desired gauge transformation is $g=\exp (X(\eta, \psi))$. If $\nabla, \phi, \eta$, and $\psi$ are $C^{\infty}$ then, applying the theorem with $k \geqq 4, X$ is $C^{2}$; elliptic regularity (Morrey, Sect. 6.8) then implies that $X$, and hence $g$, is $C^{\infty}$.

The slice theorem gives a manifold structure on the orbit space of the gauge group.

Theorem 4.2. Let $\mathscr{R} \subset \mathscr{C} \times \mathscr{E}$ be the set of regular points. Then for $k>0,2<p<4$, $\mathscr{R}_{k+1, p} / \mathscr{G}_{k+2, p}$ is a smooth Banach manifold and $\mathscr{R}_{k+1, p} \rightarrow \mathscr{R}_{k+1, p} / \mathscr{G}_{k+2, p}$ is a

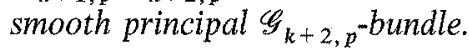

Proof. We must first show that the slice of Theorem 4.1 is globally effective, i.e. that the slice intersects each gauge orbit at most once. For this we adapt the argument of Atiyah et al. [4].

Fix $\left(\nabla_{0}, \phi_{0}\right) \in \mathscr{R}_{k+1, p}$ and let $N$ be a neighborhood of $\left(\nabla_{0}, \phi_{0}\right)$ in the slice at $\left(\nabla_{0}, \phi_{0}\right)$. Since $\mathscr{R}$ is open we may assume that $N \subset \mathscr{R}$. The orbit through any $\left(\nabla_{1}, \phi_{1}\right) \in N$ under the identification $\mathscr{C} \times \mathscr{E}=A^{1} \times \mathscr{E}$ by $(\nabla, \phi) \leftrightarrow\left(\nabla-\nabla_{1}, \phi-\phi_{1}\right)$ is $\left\{\left(g \nabla_{1} g^{-1},(g(g)-I) \phi_{1}\right) / g \in \mathscr{G}_{k+2, p}\right\}$. Under the embedding $\mathscr{G}_{e} \subset$ End $(g)$ the elements $g \in \mathscr{G}_{e}$ have pointwise constant norm $\mathcal{c}_{0}$. Let $\operatorname{End}(g)=V_{0} \oplus V_{1}$ be the orthogonal decomposition, where $V_{0}$ corresponds to the $\mathrm{Ad} G$-invariant endomorphisms and write $g=g_{0}+g_{1}$. Now $\nabla_{1}$ induces a connection on End $(g)$ and $L_{2}\left(V_{1}\right)$ decomposes into the (finite dimensional) eigenspaces of the Laplacian $\nabla_{1}^{*} \nabla_{1}$ :

$$
L_{2}\left(V_{1}\right)=\Gamma_{0}\left(V_{1}\right) \oplus \sum_{\lambda>0} \Gamma_{\lambda}\left(V_{1}\right)
$$

The map $g \mapsto(\varrho(g)-I) \phi_{1}$ induces a continuous map

$$
\mathscr{G}_{k+2, p} \cap \Gamma_{\mathrm{o}}\left(V_{1}\right) \rightarrow L_{2}(E)
$$


whose image is compact and - since $\left(\nabla_{1}, \phi_{1}\right)$ is regular - does not contain the zero section. Hence $\left\|(\varrho(g)-I) \phi_{1}\right\|_{0,2}^{2} \geqq c_{1}$ and

$$
\left\|g_{1}\right\|_{0,2}^{2} \leqq\|g\|_{0,2}^{2} \leqq c_{1}^{-1} c_{0} \cdot \operatorname{Vol} M \cdot\left\|(\varrho(g)-I) \phi_{1}\right\|_{0,2}^{2}
$$

for all $g \in \mathscr{G}_{k+2, p} \cap \Gamma_{0}\left(V_{1}\right)$. Since the elements of $\Gamma_{\lambda}\left(V_{1}\right) \lambda>0$ satisfy the Poincaré inequality we obtain

$$
\left\|g_{1}\right\|_{0,2}^{2} \leqq c_{2}\left(\left\|g \nabla_{1} g^{-1}\right\|_{0,2}^{2}+\left\|(\varrho(g)-I) \phi_{1}\right\|_{0,2}^{2}\right) \quad \forall g \in \mathscr{G}_{k+2, p} .
$$

Interpolating as above and noting the equivalence of $\|_{k, p, \nabla_{0}}$ and $\|_{k, p, V_{1}}$, this extends to

$$
\left\|g_{1}\right\|_{k+2, p, \nabla_{0}} \leqq c_{3}\left(\left\|g \nabla_{1} g^{-1}\right\|_{k+1, p, \nabla_{0}}+\left\|(\varrho(g)-I) \phi_{1}\right\|_{k+1, p, \nabla_{0}}\right) .
$$

Now if $\left(\nabla_{2}, \phi_{2}\right)=\left(g \nabla_{1} g^{-1}, \varrho(g) \phi_{1}\right) \in N$ is gauge equivalent to $\left(\nabla_{1}, \phi_{1}\right)$ then

$$
\left\|\left(\nabla_{2}-\nabla_{1}, \phi_{2}-\phi_{1}\right)\right\|_{k+1, p_{1}, \nabla_{0}} \geqq c_{3}^{-1}\left\|g_{1}\right\|_{k+2, p, \nabla_{0}}
$$

from (4.6). By the Sobolev embedding this implies (with $N$ possibly smaller) that $g_{1}$ is small in the sup norm. Decompose $g$ into simple factors $g^{i}$ so $\mathscr{G}_{e} \subset \Sigma_{i} \operatorname{End}\left(g^{i}\right)$ $=\Sigma_{i} V_{0}^{i} \oplus V_{1}^{i}$, where each $V_{0}^{i}$ is one-dimensional. We then have

$$
\left|g_{0}^{i}-I\right|=1-\left|g_{0}^{i}\right|=1-\sqrt{1-\left|g_{1}^{i}\right|^{2}} \leqq\left|g_{1}^{i}\right|
$$

and $|g-I|^{2}=\left|g_{0}-I\right|^{2}+\left|g_{1}\right|^{2} \leqq 2\left|g_{1}\right|^{2}$ pointwise. Inserting this in (4.5) and (4.6) gives

$$
\left\|\left(\nabla_{2}-\nabla_{1}, \phi_{1}-\phi_{1}\right)\right\|_{k+1, p, \nabla_{0}} \geqq c_{4}\|g-I\|_{k+2, p, \nabla_{0}} .
$$

Again making $N$ possibly smaller, (4.7) insures that $\|g-I\|_{k+2, p}$ is small enough for Theorem 4.1 to apply and we conclude that $g$ is the identity. Thus the slice is globally effective.

The proof is completed by a straightforward argument; see Mitter and Viallet [12].

\section{Gauges and Regularity}

In this section we show that solutions to the coupled field equations have the regularity properties expected for elliptic equations, specifically, that for $p>2$ an $L_{1, p}$ weak solution is $C^{\infty}$. This is basically elliptic regularity; the subtlety is that the coupled equations are elliptic only after a choice of gauge. We first reinterpret the Slice Theorem. Rather than using a connection to identify $\mathscr{C}=A^{1}$, we shall choose a point $x \in M$ and a ball $B=B(x ; r)$ around $x$ and use a fixed gauge considered as a section of the frame bundle of $E$ - to pull down connections. This identifies the space $\mathscr{C}_{B}$ of connections over $B$ with $\left.A^{1}\right|_{B}$. Let $\nabla_{0}$ be the connection corresponding to $\left.0 \in A^{1}\right|_{B}$ under this identification. Then, in terms of covariant derivatives, the original connection is $\nabla=d+\omega$, and $\nabla_{0}$ is simply exterior differentiation $d$. The Slice Theorem 4.1 (with $\phi \equiv 0$ ) yields :

Proposition 5.1. Let $\nabla$ be an $L_{k+1 . p}, k \geqq 0,2<p<4$ connection on a bundle $E$ over a 4-manifold $M$ and let $\sigma: M \rightarrow \operatorname{Frame}(E)$ be a $C^{\infty}$ gauge for $E$. Then there exists $a$ constant $\gamma>0$ depending only on $M$ such that if $\nabla=d+\omega$ and $\|\omega\|_{k+1, p}<\gamma$ in the 
gauge $\sigma$ then there is a gauge transformation $g \in \mathscr{G}_{k+2, p}$ such that $d^{*} \omega=0$ in the gauge $g \cdot \sigma$. If $\nabla$ is $C^{\infty}$ then $g$ is $C^{\infty}$.

We now have a two step program for obtaining natural gauges: (i) Choose a $C^{\infty}$ gauge around a given point $x_{0}$, and (ii) modify this to a gauge in which $d^{*} \omega=0$ using the above proposition. To accomplish (ii) it is necessary to make the $L_{k, p}$ norm of the fields small. For this we shall use the rescaling principle.

Theorem 5.2. Let $\nabla$ be an $L_{1, p} p<2<4$ connection on a domain $D \subset M^{4}$. Then there is a $C^{\infty}$ gauge $\sigma$ and a gauge transformation $g \in \mathscr{G}_{2, p}$ such that, after a constant conformal change of metric, $d^{*} \omega=0$ in a neighborhood of $0 \in D$ in the gauge $g \cdot \sigma$ and the new metric.

Proof. Given $\varepsilon>0$ choose a $C^{\infty}$ gauge in a neighborhood of $0 \in D$ and a small ball $B\left(1, \tau^{2}\right), \tau>1$, around 0 with $\|\nabla \omega\|_{0, p}<\varepsilon$ in the original scale. Enlarge $B\left(1, \tau^{2}\right)$ to the unit disk $B(\tau, 1)$ by a conformal change of metric. Since $|\omega|^{2 p}$ and $|\nabla \omega|^{p}$ $=\left|\sum e^{k} \otimes \nabla_{k} \omega\right|^{p}$ have conformal weight $2 p$ rescaling gives

$$
\|\omega\|_{0,2 p ; B(\tau, 1)}^{2 p}=\tau^{2 p-4}\|\omega\|_{0,2 p ; B\left(1, \tau^{2}\right)}^{2 p} \quad \text { and } \quad\|\nabla \omega\|_{0, p ; B(\tau, 1)}^{p}=\tau^{2 p-4}\|\nabla \omega\|_{0, p ; B\left(1, \tau^{2}\right)}^{p} .
$$

In the new metric we have, by Hölder's inequality,

$$
\begin{aligned}
\|\omega\|_{1, p ; B(\tau, 1)} & \leqq\|\sigma\|_{0, p ; B(\tau, 1)}+c\|\omega\|_{0,2 p ; B(\tau, 1)} \\
& \leqq\|\nabla \omega\|_{0, p ; B\left(1, \tau^{2}\right)}+c\|\omega\|_{0,2 p ; B\left(1, \tau^{2}\right)} \\
& \leqq(1+\mathcal{c}) \varepsilon,
\end{aligned}
$$

where $c^{2 p}$ is the volume of the unit ball in the rescaled metric which is uniformly bounded in $\tau$ for $\tau<1$. When $\varepsilon$ is sufficiently small Proposition 5.1 applies. This completes the proof.

Uhlenbeck [20] has proved the much more difficult fact that the rescaling used in this proof depends only on $\|\Omega\|_{0, p}$.

We can now prove a regularity theorem.

Theorem 5.3. Let $(\nabla, \phi) \in(\mathscr{C} \times \mathscr{E})_{1, p} p>2$ be a weak solution to the coupled YangMills equations (2.5) or (2.6). Then there is an $L_{2, p}$ gauge in which $(\nabla, \phi)$ is $C^{\infty}$.

Proof. Fix $x \in M$. By Theorem 5.2 there is an $L_{2, p}$ gauge defined in a neighborhood of $x$ such that $\nabla=d+\omega$ with $d^{*} \omega=0$ in this gauge. Expanding the field equations in this gauge, we have $\left.\left.J=D^{*} \Omega=d^{*} d \omega+\omega\right\rfloor d \omega+\frac{1}{2} \omega\right\rfloor[\omega, \omega]$. But $d d^{*} \omega=0$, so $d^{*} d \omega=\square \omega=V^{*} \nabla \omega+\operatorname{Ric}(\omega)$ by (1.18). Thus a boson field satisfies

$$
\left\{\begin{array}{l}
\left.\Delta \omega-\operatorname{Ric}(\omega)-\omega\lrcorner d \omega-\frac{1}{2} \omega\right\lrcorner[\omega, \omega]-\operatorname{Re}\langle(\nabla+\omega) \phi, \varrho() \phi\rangle=0 \\
\Delta \phi+2 \omega\lrcorner \phi+\omega\rfloor \omega(\phi)+\frac{s}{6} \phi+a|\phi|^{2} \phi+m^{2} \phi=0
\end{array}\right.
$$

weakly, where $\Delta$ is the (metric) Laplacian on functions. Applying $\mathscr{D}$ to the equation $\mathscr{D} \phi=m \phi$ and using (1.14) gives similar equations for fermion fields. These are uniformly elliptic systems, and regularity follows by standard elliptic theory (Morrey, Sect. 6.8). 


\section{Gauge Invariant Estimates}

We now begin the calculations leading to our first main estimate on solutions to the coupled field equations : the supremum of $|F|$ in a ball is bounded by the energy $\int|F|^{2}$ in a larger ball (Theorem 6.3). For this we use the DeGeorgi-Nash iteration scheme, following (Morrey, Sect. 5.3). There are, however, two complications. First, because differential equations for vector-valued functions are not directly amenable to this approach we must convert the coupled differential equations for $\phi$ and $\Omega$ into a differential inequality for the function $|F|$. Second, in order to manage the non-linearity of the resulting inequality we need a growth condition on the $L^{2}$ norm of the coefficients. This is easily verified if we assume sufficient regularity for the fields, as we shail. (In the next section, when we take up the problem of the removability of singularities the need for this growth condition will be the chief difficulty.)

All estimates in this section will be a priori, that is, we will assume (by Theorem 5.3) that the fields are $C^{\infty}$ away from an isolated singularity. We will also treat $m$ as a function rather than a constant; this will be important later, in Sect. 8, when we examine how solutions on $\mathbb{R}$ can extend to $S^{4}$.

The computations are greatly facilitated by choosing an appropriate coordinate system. Fix a point $x \in M$ and let $\left\{e_{i}\right\}$ be an orthonormal basis of $T_{x} M$. Extend the $e_{i}$ to local vector fields $e_{i}$ such that $\left(\nabla_{i} e_{j}\right)(x)=0$ for all $i, j$, and hence $\left[e_{i}, e_{j}\right](x)=0$. Let $e^{i}$ denote the 1 -form dual to $e_{i}$. The Riemannian curvature form $R$ and the bundle curvature form $\Omega=\Omega^{E}$ at $x$ are then

$$
\left\{\begin{array}{l}
R_{i j}=\nabla_{i} \nabla_{j}-\nabla_{j} \nabla_{i} \\
\Omega_{i j}^{E}=\nabla_{i}^{E} V_{j}^{E}-\nabla_{j}^{E} \nabla_{i}^{E}
\end{array}\right.
$$

Choose a local basis of sections of the adjoint bundle $g$ in the same manner: let $\left\{\sigma_{\alpha}\right\}$ be an orthonormal basis of $g_{x}$ and extend these to local sections with $\left(\nabla_{i} \nabla_{\alpha}\right)(x)=0$ for all $i, \alpha$. Let $\left\{\sigma^{\alpha}\right\}$ be the dual sections of $g^{*}$. Our calculations will be done in these frames at $x \in M$, but the final result will be manifestly independent of $x$ and the orthonormal frame.

Theorem 6.1. Let $(\phi, \Omega)$ be a solution of either the coupled fermion equations (2.5) or the coupled boson equations (2.6). Then the total field $F \in \Gamma(\mathscr{F})$ (see Definition 3.5) satisfies

$$
0 \leqq \frac{1}{2} d^{*} d|F|^{2}-\left.|d| F\right|^{2}+\mu|F|^{2}+\left(5|\Omega|+\alpha|\phi|^{2}\right)|F|^{2},
$$

where $\alpha$ is a constant of weight 0 (in the boson case) or 1 (in the fermion case) and $\mu \in \Gamma\left(L^{2}\right)$ depends only on the 1-jet of the Riemannian curvature of $\left(M, g_{0}\right)$ and either the 1-jet of $m$ (in the boson case) or the 2-jet of $m$ (in the fermion case).

Proof. We begin with the fermion case, dividing the proof into five steps.

Step 1. Apply $\mathscr{D}$ to the equation $\mathscr{D} \phi=m \phi$ to get $\mathscr{D}^{2} \phi=d m \cdot \phi+m \mathscr{D} \phi=d m \cdot \phi$ $+m^{2} \phi$ and

$$
0=\left\langle\phi, \mathscr{D}^{2} \phi\right\rangle-m^{2}|\phi|^{2}-\langle\phi, d m \cdot \phi\rangle
$$


Step 2. Differentiate the equation $\mathscr{D}^{2}=m^{2} \phi+d m \cdot \phi$ using the total covariant derivative $\boldsymbol{\nabla}$ on $V \otimes E$

$$
0=\left|\nabla_{k} \mathscr{D}^{2} \phi-\partial_{k} m^{2} \cdot \phi-m^{2}\right| \nabla_{k} \phi-\left(\nabla_{k} d m\right) \cdot \phi-d m \cdot \nabla_{k} \phi .
$$

Commuting derivatives in the leading term introduces curvature terms: $\nabla_{k} \mathscr{D}$ $=\left|\nabla_{k}\left(e^{i} \cdot \mid \nabla_{i}\right)=e^{i} \cdot\left(\left|\nabla_{i}\right| \nabla_{k}+\Omega_{k i}\right)=\mathscr{D}\right| \nabla_{k}+e^{i} \cdot \Omega_{k i}$ and

$$
\begin{aligned}
\mid \nabla_{k} \mathscr{D}^{2} & =\mathscr{D} \mid \nabla_{k} \mathscr{D}+e^{i} \cdot \bar{\Omega}_{k i} e^{j} \cdot \nabla_{j} \\
& =\mathscr{D}^{2}\left|\nabla_{k}+e^{j} \cdot\right| \nabla_{j}\left(e^{i} \cdot \bar{\Omega}_{k i}\right)+e^{i} \cdot e^{j} \cdot \bar{\Omega}_{k i} \mid \nabla_{j} \\
& =\mathscr{D}^{2}\left|\nabla_{k}-e^{j} \cdot e^{i} \cdot\left(\mid \nabla_{j} \bar{\Omega}_{k i}\right)+e^{j} \cdot e^{i} \cdot \bar{\Omega}_{k i}\right| \nabla_{j}+e^{i} \cdot e^{j} \cdot \bar{\Omega}_{k i} \mid \nabla_{j} \\
& =\mathscr{D}^{2}\left|\nabla_{k}+\sum_{i<j} e^{j} \cdot e^{i} \cdot\left(\left|\nabla_{j} \bar{\Omega}_{k i}-\right| \nabla \bar{\Omega}_{k j}\right)-\right| \nabla \bar{\Omega}_{k i}-2 \bar{\Omega}_{k i} \mid \nabla_{i} \\
& =\mathscr{D}^{2}\left|\nabla_{k}-\right| \nabla_{i} \bar{\Omega}_{k i}-2 \bar{\Omega}_{k i} \nabla_{i}
\end{aligned}
$$

(using the Bianchi identity). Here $\bar{\Omega}=R^{V} \otimes 1+1 \otimes \Omega^{E}$ is the total curvature of $V \otimes E$ so, for example, the second term is

$$
\nabla_{i} \bar{\Omega}_{k i}=\nabla_{i} R_{k i}+\nabla_{i} \Omega_{k i}^{E}=\left(D^{*} R\right)_{k}+\left(D^{*} \Omega^{E}\right)_{k} .
$$

In this we recognize the current $\left(D^{*} \Omega^{E}\right)_{k}=J_{k}$ [originally $J \in\left(\Lambda^{1} \otimes g\right)^{*}$, but here we have its image under the identification $g=g^{*}$ and the representation $\left.1 \otimes \varrho:\left(\Lambda^{1}\right)^{*} \otimes g \rightarrow\left(\Lambda^{1}\right)^{*} \otimes E \otimes E^{*}\right]$. Combining (6.3), (6.4), and (6.5), taking the inner product with $\mid \nabla_{k} \phi$, and summing on $k$ gives

$$
\begin{aligned}
0= & \left\langle\mid \nabla \phi, \mathscr{D}^{2}(\mid \nabla \phi)\right\rangle-\left\langle\mid \nabla \phi,\left[\left(D^{*} R\right)_{k}+J_{k}\right] \cdot \phi\right\rangle-2\left\langle\left|\nabla_{k} \phi,\left(R_{k i}+\Omega_{k i}^{E}\right)\right| \nabla_{i} \phi\right\rangle \\
& -\partial_{k} m^{2}\left\langle\mid \nabla_{k} \phi, \phi\right\rangle-\left.m^{2}|| \nabla \phi\right|^{2}-\left\langle\left|\nabla_{k} \phi,\left(\mid \nabla_{k} d m\right) \cdot \phi+d m \cdot\right| \nabla_{k} \phi\right\rangle .
\end{aligned}
$$

Step 3. Differentiate the second field equation $D^{*} \Omega=J$. With the Bianchi identity $D \Omega=0$, this gives

and

$$
\begin{aligned}
\left(D D^{*}+D^{*} D\right) \Omega & =D J=-\frac{1}{2} D\left[\left\langle\phi, e^{k} \cdot \varrho\left(\sigma^{\alpha}\right) \sigma_{\alpha} \otimes e^{k}\right]\right. \\
& =-\frac{1}{2} \operatorname{Re}-\frac{1}{2} \operatorname{Re} \Sigma\left\langle\nabla_{i} \phi, e^{k} \cdot \varrho\left(\sigma^{\alpha}\right) \phi\right\rangle \sigma_{\alpha} \otimes e^{i} \wedge e^{k}
\end{aligned}
$$

$$
0=\left\langle\Omega, \mathscr{D}^{2} \Omega\right\rangle+\frac{1}{2} \operatorname{Re}\left\langle\nabla_{i} \phi, e^{k} \cdot \Omega_{i k}(\phi)\right\rangle .
$$

Step 4. Combine (6.2), (6.6), and (6.7) into a single equation for $F=\Omega$ $+\tau^{-1} \mid \nabla \phi+\tau \phi:$

$$
\begin{aligned}
0= & \left\langle F, \mathscr{D}^{2} F\right\rangle-m^{2}\left(|\tau \phi|^{2}+\left.\left|\tau^{-1}\right| \nabla \phi\right|^{2}\right)-\langle\tau \phi, d m \cdot \tau \phi\rangle-\left\langle\tau^{-1}\left|\nabla_{k} \phi, d m \cdot \tau^{-1}\right| \nabla_{k} \phi\right\rangle \\
& -\left\langle\tau^{-1} \mid \nabla_{k} \phi, \tau^{-2}\left[\left(D^{*} R\right)_{k}+J_{k}\right] \cdot \tau \phi\right\rangle-2\left\langle\tau^{-1}\left|\nabla_{k} \phi,\left(R_{k i}+\Omega_{k i}^{E}\right) \tau^{-1}\right| \nabla_{i} \phi\right\rangle \\
& -\tau^{-2} \partial_{k} m^{2}\left\langle\tau^{-1} \mid \nabla_{k} \phi, \tau \phi\right\rangle-\tau^{-2}\left\langle\tau^{-1} \nabla_{k} \phi,\left(\mid \nabla_{k} d m\right) \cdot \tau \phi\right\rangle+\frac{1}{2} \operatorname{Re}\left\langle\tau \mid \nabla_{i} \phi, e^{k} \cdot \Omega_{i k}(\tau \phi)\right\rangle .
\end{aligned}
$$

The leading term can be expanded according to the Weitzenböck formulae (1.14), (1.19) for $\mathscr{D}^{2}$ on the spinors $\phi, \nabla_{k} \phi$ and on the $g$-valued 2-form $\Omega$

$$
\begin{aligned}
\left\langle F, \mathscr{D}^{2} F\right\rangle= & \left\langle F,-\left|\nabla_{i}\right| \nabla_{i} F\right\rangle+\frac{s}{4}|F|^{2}+\frac{s}{12}|\Omega|^{2}-\langle\Omega, W(\Omega)\rangle \\
& +\left\langle\tau \phi, e^{i} \cdot e^{j} \cdot \Omega_{i j}^{E}(\tau \phi)\right\rangle+\left\langle\tau^{-1} \mid \nabla_{k} \phi, e^{i} \cdot e^{j} \cdot \Omega_{i j}^{E}\left(\tau^{-1} \mid \nabla_{k} \phi\right)\right\rangle+\langle\Omega,[\Omega, \Omega]\rangle .
\end{aligned}
$$


Let $\mathscr{R}$ be the bundle map $\mathscr{F} \rightarrow \mathscr{F} \otimes L^{2}$ defined by

$$
\mathscr{R}(F)=\frac{s}{4} F+\frac{s}{12} \Omega^{E}-W\left(\Omega^{E}\right)-\tau^{-2} D^{*} R \otimes \tau \phi-2 R_{k i} \cdot \tau^{-1} \mid \nabla_{i} \phi \cdot e^{k}
$$

(regarding $\tau^{-2} D^{*} R \in T \otimes g \otimes L^{2}$ as a map $V^{2} \otimes E \rightarrow T \otimes V^{4} \otimes E$ ). Then $\mathscr{R}$ depends on the 1 -jet of the Riemannian curvature of $M$ and is bounded by a constant $\varrho$ of conformal weight 2 . Now substitute into (6.8), multiply by -1 , and estimate, using

$$
\begin{aligned}
\left\langle\tau^{-1} \mid \nabla_{k} \phi, \tau^{-2} J_{k}(\tau \phi)\right\rangle & \leqq \frac{1}{2} \tau^{-2}\left|\left\langle\phi, e^{k} \cdot \varrho() \phi\right\rangle\left\langle\tau^{-1} \mid \nabla_{k} \phi, \varrho() \tau \phi\right\rangle\right| \\
& \leqq \tau^{-2}|\phi|^{2} \cdot|F|^{2}
\end{aligned}
$$

[cf. (2.5)]. The result is the more manageable inequality

$$
0 \leqq\left\langle F,\left|\nabla_{i}\right| \nabla_{i} F\right\rangle+\mu|F|^{2}+\left(5|\Omega|+\tau^{-2}|\phi|^{2}\right)|F|^{2},
$$

where $\mu=\left[\varrho+m^{2}+|d m|+\tau^{-2}\left|d m^{2}\right|+\tau^{-2}|2 d m|\right]$ is a function of conformal weight 2.

Step 5. Because $\mathbb{V}$ is compatible with the metrics on $V \otimes E$ and $A^{2} \otimes g$,

$$
d^{*} d|F|^{2}=2 d^{*}\left[\operatorname{Re}\left\langle F, \mid \nabla_{i} F\right\rangle e^{i}\right]=2 \operatorname{Re}\left[\langle|\nabla F,| \nabla F\rangle+\left\langle F,\left|\nabla_{i}\right| \nabla_{i} F\right\rangle\right]
$$

so $\left\langle F,\left|\nabla_{i}\right| \nabla_{i} F\right\rangle=\frac{1}{2} d^{*} d|F|^{2}-\left.|| F F\right|^{2} \leqq \frac{1}{2} d^{*} d|F|^{2}-|d| F \|^{2}$ by Kato's inequality. Putting this in (6.9) completes the proof in the fermion case.

The boson case is completely analogous so we will be brief.

Step 1. From $0=\left|V^{*}\right| \nabla \phi-\frac{s}{6} \phi-a|\phi|^{2} \phi-m^{2} \phi$ we obtain $0=\left\langle\phi,-\left|V_{i}\right| \nabla \phi\right\rangle-\frac{s}{6}|\phi|^{2}$ $-a|\phi|^{4}-m^{2}|\phi|^{2}$

Step 2. On $E$-valued scalars we have $\left|\nabla^{*}\right| \nabla=D^{*} D$, while

$$
\mid \nabla_{k} D=\nabla_{k}\left(e^{i} \wedge \mid \nabla_{i}\right)=e^{i} \wedge\left(\mid \nabla_{i} \nabla_{k}+\bar{\Omega}_{k i}\right)=D \nabla_{k}+e^{i} \wedge \bar{\Omega}_{k i}
$$

and similarly $\left.\nabla_{k} D^{*}=D^{*} \mid \nabla_{k}-e_{j}\right\lrcorner \bar{\Omega}_{k j^{*}}$ Hence

$$
\begin{aligned}
\nabla_{k} D^{*} D & =D^{*} D\left|V_{k}-2 \bar{\Omega}_{k i} e_{j} e^{i} \wedge \nabla_{j}-\right| \nabla_{i} \bar{\Omega}_{k i} \\
& =D^{*} D\left|\nabla_{k}-2\left(R_{k i}+\Omega_{k i}^{E}\right)\right| \nabla_{i}-\left(D^{*} R\right)_{k}-J_{k}
\end{aligned}
$$

$\left(J_{k}\right.$ is the boson current), and $0=\left\langle\nabla_{k} \phi, \mid \nabla_{k}\left(\left|V^{*}\right| \nabla \phi-\frac{s}{6} \phi-a|\phi|^{2} \phi-m^{2} \phi\right)\right\rangle$ becomes

$$
\begin{aligned}
0= & \left\langle\left|\nabla_{k} \phi,-\right| \nabla_{i} \mid \nabla_{i}\left(\mid V_{k} \phi\right)\right\rangle-2\left\langle\left|\nabla_{k} \phi,\left(R_{k i}+\Omega_{k i}^{E}\right)\right| \nabla_{i} \phi\right\rangle \\
& -\left\langle\mid \nabla_{k} \phi,\left(\left(D^{*} R\right)_{k}+J_{k}\right) \phi\right\rangle-\left.\left(\frac{s}{6}+a|\phi|^{2}+m^{2}\right)|| \nabla \phi\right|^{2} \\
& -\hat{\partial}_{k}\left(\frac{s}{6}+a|\phi|^{2}+m^{2}\right)\left\langle\mid \nabla_{k} \phi, \phi\right\rangle
\end{aligned}
$$


Step 3. From $D^{*} \Omega=J$ we obtain $0=\langle\Omega, \square \Omega-D J\rangle$. Here $\square \Omega$ can be expanded using (1.19) while

$$
\begin{aligned}
-\langle\Omega, D J\rangle & =\operatorname{Re}\left\langle\Omega, D\left(\left\langle\mid \nabla_{k} \phi, \varrho() \phi\right\rangle e^{k}\right)\right\rangle \\
& =\operatorname{Re}\left[\left\langle\nabla_{i} \mid \nabla_{k} \phi, \Omega_{i k}(\phi)\right\rangle+\left\langle\mid \nabla_{k} \phi, \Omega_{i k}\left(\mid \nabla_{i} \phi\right)\right\rangle\right] \\
& =\frac{1}{2}|\Omega(\phi)|^{2}+\left\langle\mid \nabla_{k} \phi, \Omega_{i k}\left(\mid \nabla_{i} \phi\right)\right\rangle .
\end{aligned}
$$

Step 4. Set $F=\Omega+\nabla \phi+\tau^{2} \phi \in \Gamma(\mathscr{F})$, define $\mathscr{R}:: \mathscr{F} \rightarrow \mathscr{F} \otimes L^{2}$ by

$$
\mathscr{R}(F)=\frac{s}{6} F-\frac{s}{2} \Omega-W(\Omega)+2 R_{k i} \mid \nabla_{i} \phi-\frac{1}{6} \tau^{-2} d s \otimes \tau^{2} \phi+\tau^{-2} D^{*} R \otimes \tau^{2} \phi,
$$

and let $\varrho$ be an upper bound for $\mathscr{R}$. Add the results of Steps 1,2, and 3, multiply by -1 and estimate, noting that

$$
\left\langle\mid \nabla_{k} \phi, J_{k}(\phi)\right\rangle \leqq|\langle\mid \nabla \phi, \varrho() \phi\rangle|^{2} \leqq\left.|\phi|^{2}|| \nabla \phi\right|^{2}
$$

and

$$
\partial_{k}|\phi|^{2} \cdot\left\langle\mid \nabla_{k} \phi, \phi\right\rangle \leqq 2 \cdot|\langle\mid \nabla \phi, \phi\rangle|^{2} \leqq\left. 2 \cdot|\phi|^{2}|| \nabla \phi\right|^{2}
$$

The result is

$$
0 \leqq\left\langle F,\left|\nabla_{i}\right| \nabla_{i} F\right\rangle+\mu^{\prime}|F|^{2}+5|\Omega| \cdot|F|^{2}+(1+3 a+|d a|)|\phi|^{2} \cdot|F|^{2},
$$

where $\mu^{\prime}=\left(\varrho+m^{2}+\tau^{-2} d m^{2}\right)$ has weight 2. The proof is completed by Step 5 above.

Proposition 6.2. Let $F$ be a $C^{\infty}$ field which satisfies the differential inequality (6.1) on a ball $B_{r} \subset M$ of radius $r$. Then for any $\eta \in C_{0}^{\infty}\left(B_{r}\right)$ and $p>\frac{1}{2}$

$$
\begin{aligned}
\int_{B_{r}}\left|d\left(\eta|F|^{p}\right)\right|^{2} \leqq & C(p) \int_{B_{r}}\left(\left|d^{*} d \eta^{2}\right|+4|d \eta|^{2}\right)|F|^{2 p} \\
& +c^{\prime} \beta(r) p C(p) \int_{B_{r}}\left|d\left(\eta \cdot|F|^{p}\right)\right|^{2}
\end{aligned}
$$

for some constant $c^{\prime}$, where $C(p)=p / 2 p-1$ and $\beta^{2}$ is the conformally invariant integral

$$
\beta^{2}(r)=\int_{B_{r}}|\mu|^{2}+5|\Omega|^{2}+\alpha^{2}|\phi|^{4} .
$$

Proof. Set $b=|\mu|+5|\Omega|+\alpha|\phi|^{2}$ and evaluate (6.1) on the test function $\eta^{2} f^{2 p-2}$, where $f=|F|$ (technically, take $f=|F|+\varepsilon$ and let $\varepsilon \rightarrow 0$ at the end). Integrate by parts and simplify using $d f^{2 p}=p f^{2 p-2} d f^{2}$, etc.

$$
\begin{aligned}
0 & \leqq-\frac{1}{2} \int d\left(\eta^{2} f^{2 p-2}\right) d f^{2}-\int \eta^{2} f^{2 p-2}|d f|^{2}+\int b \eta^{2} f^{2 p} \\
& \leqq-\frac{1}{2 p} \int d \eta^{2} \cdot d f^{2 p}-\frac{2 p-2}{p^{2}} \int \eta^{2}\left|d f^{p}\right|^{2}-\frac{1}{p^{2}} \int \eta^{2}\left|d f^{p}\right|^{2}+\int b \eta^{2} f^{2 p} \\
& \leqq \frac{1}{2 p} \int\left|d^{*} d \eta^{2}\right| f^{2 p}-\frac{2 p-1}{p^{2}} \int \eta^{2}\left|d f^{p}\right|^{2}+\int b \eta^{2} f^{2 p}
\end{aligned}
$$


Transfer the middle term to the left-hand side, multiply by $\frac{2 p^{2}}{2 p-1}$ and note that $\frac{p}{2 p-1}>\frac{1}{2}$ and $\left|d\left(\eta f^{p}\right)\right|^{2} \leqq 2|d \eta|^{2} f^{2 p}+2 \eta^{2}\left|d f^{p}\right|^{2}$. This gives

$$
\int\left|d\left(\eta f^{p}\right)\right|^{2} \leqq \frac{p}{2 p-1} \int\left(\left|d^{*} d \eta^{2}\right|+4|d \eta|^{2}\right) f^{2 p}+\frac{2 p^{2}}{2 p-1} \int b \eta^{2} f^{2 p},
$$

and the last term is bounded by the Hölder and Poincaré-Sobolev inequalities

$$
\int b \eta^{2} f^{2 p} \leqq\left(\int b^{2}\right)^{1 / 2}\left(\int\left(\eta f^{p}\right)^{4}\right)^{1 / 2} \leqq c^{\prime} \beta(r) \int\left|d\left(\eta f^{p}\right)\right|^{2} .
$$

Theorem 6.3. Let $(\phi, \Omega)$ be a $C^{\infty}$ solution on a ball $B_{2 R} C M$ to either

(i) the coupled fermion equations (2.5) with $m \in L_{2, p}\left(B_{2 R}\right)$ for some $p>2$,

(ii) the coupled boson equations (2.6) with $m^{2} \in L_{1, p}\left(B_{2 R}\right)$ for some $p>2$.

Then there is a constant $C$ of conformal weight zero such that for all $x \in B_{R}$ and $r \leqq R$

$$
|F(x)|^{2} \leqq C r^{-4} \int_{B_{R+r}}|F(y)|^{2} d y .
$$

Proof. Since $F$ and the Riemannian curvature of $M$ are $C^{\infty}$ the hypotheses imply that $\mu \in L_{p}\left(B_{2 R}\right)$ for some $p>2$ and, by Hölder's inequality, that $\beta(r) \leqq c_{1} r^{\delta}$ for some $\delta>0$.

Fix $p \geqq 1$ and set $r_{p}=\left(2 c_{1} c^{\prime} p C(p)\right)^{-1 / \delta}$. Then whenever $r \leqq r_{p} \beta\left(r_{p}\right) \leqq\left(2 c^{\prime} p C(p)\right)^{-1}$ and we can move the last term of (6.10) to the left-hand side. Choosing $\eta \in C_{0}^{\infty}\left(B_{2 r}\right)$ with $\eta \equiv 1$ on $B_{r}, 0 \leqq \eta \leqq 1,|d \eta| \leqq 2 / r$ and $\left|d^{*} d \eta^{2}\right| \leqq 2 / r^{2}$ gives

$$
\left.\left.\int_{B_{r}}|d| F\right|^{p}\right|^{2} \leqq \int_{B_{2 r}}\left|d\left(\eta \cdot|F|^{p}\right)\right|^{2} \leqq c r^{-2} \int_{B_{2 r}}|F|^{p} \quad \forall r \leqq r_{p}
$$

for some constant $c$. Now given a ball $B_{R}$ of large radius we can cover it with balls of radius $r<r_{p}$ and use the above and the Poincaré-Sobolev inequality to obtain (cf. Morrey, p. 136)

$$
\left(\int_{B_{R}}|F|^{4 p}\right)^{1 / 2} \leqq c(p C(p))^{2 / \delta} \cdot r^{-2} \int_{B_{R+r}}|F|^{2 p}, \quad 0 \leqq r \leqq R, \quad p \geqq 1 .
$$

The inequality now follows by iteration (Morrey, Theorem 5.3.1), and counting conformal weights we see that $C$ is weightless.

Theorem 6.3 is immediately applicable to the problem of the behavior of fields around a singularity.

Corollary 6.4. Let $(\phi, \Omega)$ be a solution to the coupled field equations which is $C^{\infty}$ in a neighborhood of a point $p \in M$ but is undefined at $p$. Assume that the energy $\alpha(r)$ is finite on some ball $B(p ; r)$ and that the mass $m$ satisfies the hypotheses of Theorem 6.3 on $B(p ; r)$. Take $p$ as the origin and let $\varepsilon>0$ be given. Then $(\phi, \Omega)$ is conformally equivalent to a solution on $B_{2}$ with

$$
|\Omega,(x)|,|\nabla \phi(x)| \leqq|F(x)| \leqq C|x|^{-2} \cdot E(2|x|) \leqq C \varepsilon|x|^{-2}
$$

for each $x \in B_{1}-\{0\}$. Moreover, $|\phi|=O\left(\frac{1}{r}\right)$ at the origin. 
Proof. By rescaling we may assume that $(\phi, \Omega)$ is defined on $B_{2}$ and that $E(2) \leqq \varepsilon$. For $x \in B_{1}-\{0\}$ set $r=\frac{1}{2}|x|$. Then $B_{2 r} \subset B_{2}-\{0\}$ and $F$ is $C^{\infty}$ in $B_{r}$. By Theorem 6.3

$$
|F(x)|^{2} \leqq C|x|^{-4} \int_{B(x, 2 r)}|F|^{2} \leqq C|x|^{-4} \cdot E^{2}(2|x|)
$$

for $x \in B_{1}-\{0\}$. This gives the first statement and the second follows by noting that $|d| \phi\|\leqq\| \nabla \phi \mid$ and integrating inward from the unit sphere.

\section{An Energy Growth Rate}

We shall now focus attention on $C^{\infty}$ solutions of the coupled field equations with finite energy and with an isolated singularity. We have just derived one fact about such fields: $|\Omega|$ and ||$\nabla \phi \mid$ grow as $O\left(r^{-2}\right)$ at the singularity. We also have the regularity Theorem 5.3 at our disposal, but this requires that the field be $L_{1, p}$ for some $p>2$. What is needed is a growth rate of the form

$$
\int_{B_{r}}|F|^{2} \leqq c r^{\delta}
$$

for some $c$ and $\delta>0$, where the ball $B_{r}$ is centered on the singularity. This section is devoted to establishing such an estimate.

Although the growth estimate involves only gauge independent quantities the proof requires a specific choice of gauge - a trivialization of the principal bundle $P$. For this we rely on a theorem of Uhlenbeck which provides a trivialization around the singularity and includes rather specific information about the connection and curvature forms in this gauge. Integration by parts in this gauge, first for the field $\phi$ and then for the curvature form $\Omega$, results in an inequality for the energy integral $E(r)$. By the rescaling process this becomes a differential inequality and integrating gives the desired growth rate for $E(r)$.

Throughout this section we assume the fields are $C^{\infty}$ away from an isolated singular point, taken as the coordinate origin. We shall also assume that the mass coefficient $m$ satisfies the hypotheses of Theorem 6.3. The rescaling process allows us to work in an arbitrarily small neighborhood of the singularity, conformally enlarged to the unit ball. Consequently, we expediently ignore the curvature of the base manifold. This simplification does not essentially affect the theorems.

A few words on notation. The letter $c$ will be used for a universal constant (depending on Sobolev norms, the volume of the unit ball, etc.). Its value will be continually updated so, for example, when $c$ is multiplied by 2 the result is immediately renamed $c$. We will need the energy integral $E(\tau, r)$ defined by (3.5). After fixing a scale we will denote $E(\tau, r)$ by $E$, or replace it in the estimates by an upper bound $\varepsilon$. We will frequently use the evident inequalities $\|\Omega\|_{02},\|\nabla \phi\|_{02}$ $\leqq E \leqq \varepsilon$ and the Sobolev inequality $\|\phi\|_{04}^{2} \leqq c E^{2} \leqq c \varepsilon^{2}$. All Sobolev norms are taken on the unit ball.

The starting point for our gauge dependent estimates is the following theorem of Uhlenbeck.

Theorem 7.1 (Uhlenbeck [19]). Let $\omega$ be a $C^{\infty}$ connection form on $B_{2}-\{0\}$ with curvature $\Omega$. Then there is a constant $\kappa$ such that if $|\Omega(x)| \cdot|x|^{2} \leqq \varepsilon<\kappa$ on $B_{1}-\{0\}$ 
then there exists a "broken harmonic gauge" on $B_{1}-\{0\}$ whose properties we now describe. Set

$$
\begin{aligned}
& A_{i}=\left\{x\left|2^{-i} \leqq\right| x \mid \leqq 2^{-i+1}\right\} \\
& S_{i}=\left\{x|| x \mid=2^{-i}\right\}=\text { inside boundary of } A_{i}
\end{aligned}
$$

The broken harmonic gauge is $C^{\infty}$ on each $A_{i}$ and the gauges from $A_{i}$ and $A_{i+1}$ agree on $S_{i^{*}}$ Write

$$
\left.\begin{array}{l}
\omega_{i}=\left.\omega\right|_{A_{i}} \\
\Omega_{i}=\left.\Omega\right|_{A_{i}}
\end{array}\right\} \text { expressed in the gauge on } A_{i}
$$

Then $\left\{\omega^{i}\right\}$ and $\left\{Q^{i}\right\}$ satisfy

(a) $\left.\omega_{\theta}^{i}\right|_{s_{i}}=\left.\omega_{\theta}^{i+1}\right|_{s_{i}}, \omega_{\theta}=$ tangential components of $\omega$,

(b) $d^{*} \omega^{i}=0$

(c) $\bar{d}^{*} \omega_{\theta}^{i}=0$ on $S_{i}$ and $S_{i-1}$, where ${ }^{-}$denotes restriction to the sphere,

(d) $\int_{S_{i}} \omega_{r}^{i}=\int_{S_{i-1}} \omega_{r}^{i}=0, \omega_{r}=$ radial component of $\omega$,

and there exist constants $b, \lambda>0$ such that

(e) $\left\|\omega^{i}\right\|_{\infty} \leqq 2^{-i}\|\Omega\|_{\infty} \leqq 2^{i} b \varepsilon$

(f) $\int_{A_{i}}\left|\omega^{i}\right|^{2} \leqq\left(\lambda-b^{2} \varepsilon^{2}\right)^{-1} \cdot 2^{-2 i} \int_{A_{i}}\left|\Omega^{i}\right|^{2}$.

This theorem is proved in (Uhlenbeck [19]). In outline, the construction of the gauge proceeds as follows. Choose a basis for $g$ at the north and south poles of $S_{1}$ and extend these by parallel translation along polar geodesics. These gauge meet at the equator and one shows that the transition functions can be made small. These transition functions can be spread out away from the equator, resulting in a $C^{\infty}$ gauge over $S_{1}$. The Slice Theorem then gives (c) on $S_{1}$ and, by the same process, on each $S_{i}$. Now repeat the procedure to fill in between the $S_{1}$ : we have a gauge on $S_{i}$ which extends inward by parallel translation and one on $S_{i+1}$ which extends outward; these meet in the middle of $A_{i}$ and the transition functions are small. Spread out the transition functions and apply the Slice Theorem; this gives (b). Statements (a) and (d) are inherent in this process and (e) and (f) are consequences of (a)-(d) and the curvature hypothesis.

Remarks. 1. Note that such a gauge can be chosen for any connection; it is not necessary that $\Omega$ satisfy the field equations.

2. The broken harmonic gauge trivializes the principal bundle $P$ and therefore trivializes all associated bundles $E$ over $B_{1}$.

Since we are assuming that the coefficient $m$ satisfies the hypotheses of Theorem 6.3, we can apply Corollary 6.4 to obtain a field on $B_{2}-\{0\}$ satisfying the hypotheses of Theorem 7.1. Hence there exists a broken harmonic gauge.

In the subsequent theorems this gauge is used as a "reference frame" in which to observe the field $\phi$; we will express $\omega$ and $\phi$ in the broken harmonic gauge and use estimates (a)-(f) to argue that the covariant derivatives $\nabla \phi$ of $\phi$ and $\Omega$ of $\omega$ are essentially the same as $\nabla \phi$ and $\nabla \omega$. In doing this we may assume, by the rescaling principle, that $\varepsilon$ is as small as required. 
As an example of a theorem in this vein we have

Lemma 7.2. In the broken harmonic gauge the connection form $\omega$ on the unit ball $B$ and the unit sphere $S$ satisfies

(a) $\int_{B}|\omega|^{4} \leqq c \varepsilon^{2} \cdot \int_{B}|\Omega|^{2} \leqq c \varepsilon^{4}$,

(b) $\int_{B}^{B}\left|\omega_{\theta}\right|^{4} \leqq c \varepsilon^{2} \cdot \int_{B}^{B}\left|\Omega_{\theta \theta}\right|^{2}, \omega_{\theta}=$ tangential components of $\omega$ for some constant $c$ whenever $\varepsilon$ is sufficiently small.

Proof. From Theorem 7.1e, f,

$$
\int_{A_{i}}|\omega|^{4} \leqq\left\|\omega^{i}\right\|_{\infty}^{2} \int_{A_{i}}\left|\omega^{i}\right|^{2} \leqq\left(2^{i} b \varepsilon\right)^{2}\left(\lambda-b^{2} \varepsilon^{2}\right)^{-1} 2^{-2 i} \int_{A_{i}}\left|\Omega^{i}\right|^{2} \leqq c \varepsilon^{2} \int_{A_{i}}|\Omega|^{2}
$$

Summing on $i$ gives statement (a). By Theorem 7.1c $\vec{d}^{*} \omega_{\theta}=0$ on $S$, i.e., $\omega$ is a coclosed 1-form on $S^{3}$. Let $\lambda^{\prime}$ be the first eigenvalue of the Laplacian on such forms $\left(\lambda^{\prime} \neq 0\right.$ by Hodge theory). Then $\lambda^{\prime} \int\left|\omega_{\theta}\right|^{2} \leqq \int\left|d \omega_{\theta}\right|^{2}$ on $S$ and $\|\omega\|_{\infty} \leqq b \varepsilon$ by Theorem $7.1 \mathrm{e}$, so

$$
\int\left|\Omega_{\theta \theta}\right|^{2}=\int\left|d \omega_{\theta}+\frac{1}{2}\left[\omega, \omega_{\theta}\right]\right|^{2} \geqq \int\left|d \omega_{\theta}\right|^{2}-\|\omega\|_{\infty}^{2} \int\left|d \omega_{\theta}\right|^{2}-\|\omega\|_{\infty}^{2} \int\left|\omega_{\theta}\right|^{2} \geqq \frac{\lambda^{\prime}}{2} \int\left|\omega_{\theta}\right|^{2}
$$

when $\varepsilon$ is sufficiently small. The desired inequality for $\omega_{\theta}$ is then

$$
\int_{S}\left|\omega_{\theta}\right|^{4} \leqq\|\omega\|_{\infty}^{2} \int_{S}\left|\omega_{\theta}\right|^{2} \leqq 2\left(\lambda^{\prime}\right)^{-1} b^{2} \varepsilon^{2} \cdot \int_{S}\left|\Omega_{\theta \theta}\right|^{2}
$$

The next lemma shows that the Sobolev $L_{1,2}$ norm based on $\nabla$ is essentially equivalent to the one based on $\nabla$ in the broken harmonic gauge. Before stating it we introduce some notation which will facilitate changes from $\nabla$ to $\nabla$ and allow us to deal with the fermion and boson equations simultaneously.

According to the Weitzenböck formula, the fermion equation $\mathscr{D}^{2} \phi=m^{2} \phi$ $+d m \cdot \phi$ (cf. Theorem 6.1) is $\left|\nabla^{*}\right| \nabla \phi=m^{2} \phi+d m \cdot \phi-\frac{s}{4} \phi-\sum e^{i} \cdot e^{j} \cdot \Omega_{i j}(\phi)$ and, since $d^{*} \omega=0$ by Theorem $7.1 \mathrm{~b}$

$$
\left.\left.\nabla^{*} \nabla \phi=(\mid \nabla-\omega)^{*}(\mid \nabla-\omega) \phi=\left|\nabla^{*}\right| \nabla \phi-2 \omega\right\lrcorner \mid \nabla \phi+\omega\right\lrcorner \omega(\phi) .
$$

Thus $\phi$ satisfies

$$
\left.\left.\nabla^{*} \nabla \phi=m^{2} \phi+d m \cdot \phi-\frac{s}{4} \phi-\sum e^{i} \cdot e^{j} \cdot \Omega_{i j}(\phi)-2 \omega\right\rfloor \mid \nabla \phi+\omega\right\lrcorner \omega(\phi)
$$

in the broken harmonic gauge. Similarly, a boson field satisfies

Set

$$
\left.\left.\nabla^{*} \nabla \phi=a|\phi|^{2} \phi+m^{2} \phi-\frac{s}{6} \phi-2 \omega\right\lrcorner \mid \nabla \phi+\right\lrcorner \omega(\phi) .
$$

$$
\left\{\begin{array}{l}
M(\phi)=m^{2} \phi-\frac{s}{6} \phi+\left(d m \cdot \phi-\frac{s}{12} \phi\right) \\
Q(\phi)=2 \omega\lrcorner \mid \nabla \phi+\omega\lrcorner \omega(\phi) \\
\Omega(\phi)=\left(\sum e^{i} \cdot e^{j} \cdot \Omega_{i j}(\phi)\right) \\
H(\phi)=a|\phi|^{2} \phi
\end{array}\right.
$$


[the terms in parentheses occur only in the fermion case and the Higgs potential $H(\phi)$ occurs only in the boson case]. With this notation $\left.|| \nabla \phi\right|^{2}=|\nabla \phi+\omega \phi|^{2}=|\nabla \phi|^{2}+\langle\phi, Q(\phi)\rangle$ and $\phi$ satisfies

$$
\nabla^{*} \nabla \phi=(M+H-Q-\Omega) \phi \text {. }
$$

Lemma 7.3. There is a constant $c$ such that whenever $\varepsilon$ is sufficiently small

$$
\int_{B}|\nabla \phi|^{2}-c \varepsilon^{2} E^{2} \leqq\left.\int_{B}|| \nabla \phi\right|^{2} \leqq \int_{B}|\nabla \phi|^{2}+c \varepsilon^{2} E^{2}
$$

in the broken harmonic gauge.

Proof. We have $\|\left.\nabla \phi\right|^{2}=|\nabla \phi|^{2}+\langle\phi, Q(\phi)\rangle$, while $\langle\langle\phi, Q(\phi)\rangle|\leqq 2| \omega|\cdot| \phi|\cdot||\nabla \phi|$ $+|\phi|^{2} \cdot|\omega|^{2}$ can be estimated using Lemma 7.2a:

$$
\int_{B}|\langle\phi, Q(\phi)\rangle| \leqq 2\|\omega\|_{04} \cdot\|\phi\|_{04} \cdot\|\mid \nabla \phi\|_{02}+\|\phi\|_{04}^{2} \cdot\|\omega\|_{04}^{2} \leqq c \varepsilon^{2} E^{2} .
$$

Equation (7.1) shows that to highest order the components of the field $\phi$ are harmonic functions. We next note an interesting fact about finite energy harmonic functions in four dimensions: the $L_{2}$ norm of the tangential derivatives dominates the norm of the radial derivatives.

Lemma 7.4. Let $f$ be a harmonic function on $B_{1}-\{0\} \subset \mathbb{R}^{4}$ with $\int_{B}|\nabla f|^{2}$ finite. Then

$$
\int_{B_{1}}\left|\nabla_{r} f\right|^{2} \leqq \int_{B_{1}}\left|\nabla_{\theta} f\right|^{2}
$$

Proof. Expand $f$ in spherical harmonics $\left\{\sigma_{n}\right\}: f=\sum a_{n} \sigma_{n} r^{n}$. Because the $\sigma_{k}$ are orthonormal

$$
\int_{B_{1}}\left|\nabla_{r} f\right|^{2}=\int_{0}^{1} \sum n^{2}\left(a_{n}\right)^{2} r^{2 n-2} \cdot r^{3} d r \leqq \int_{B_{1}}|\nabla f|^{2} .
$$

By hypothesis the right-hand side is finite and hence the spherical harmonic expansion of $f$ involves only non-negative powers of $r$. In four dimensions $\nabla_{r} \nabla_{r}=r^{-3} \nabla_{r}\left(r^{3} \nabla_{r}\right)$, so $\nabla_{r} \nabla_{r} f=\sum n(n+2) a_{n} \sigma_{n} r^{n-2}$. On the sphere of radius $r$

while

$$
\int_{S_{r}}\left|\nabla_{r} f\right|^{2}=\sum n^{2}\left(a_{n}\right)^{2} \cdot r^{2 n-2} \cdot r^{3},
$$

Thus

$$
\int_{S_{r}} f \cdot \nabla_{r} \nabla_{r} f=\sum n(n+2)\left(a_{n}\right)^{2} r^{2 n-2} \cdot r^{3} .
$$

$$
\int_{S_{r}} f \cdot \nabla_{r} V_{r} f-\left|V_{r} f\right|^{2}=\sum_{n>0} 2 n\left(a_{n}\right)^{2} r^{2 n-1} \geqq 0,
$$

and integrating by parts

$$
\int_{S_{r}}\left|\nabla_{\theta} f\right|^{2}=-\int_{S_{r}} f \cdot \nabla_{\theta} \nabla_{\theta} f=\int_{S_{r}} f \cdot \nabla_{r} \nabla_{r} f \geqq \int_{S_{r}}\left|\nabla_{r} f\right|^{2} .
$$

The lemma follows by integrating over $r$.

Of course the field $\phi$ is not harmonic in the broken gauge so Lemma 7.4 does not directly apply to it. However, 
Lemma 7.5. Let $\phi$ be a solution of Eq. (7.1) over the flat unit ball. Assume $M \in L_{2}(B)$. Then if $\varepsilon$ is sufficiently small

$$
\int_{B}\left|\nabla_{r} \phi\right|^{2} \leqq 2 \int_{B}\left|\nabla_{\theta} \phi\right|^{2}+c \varepsilon^{2} E^{2}+c \varepsilon^{2}\|M\|_{02}^{2}
$$

when $\phi$ is expressed in the broken harmonic gauge.

Proof. The field $\phi$ satisfies $-\Delta \phi=e(\phi)$, where $e(\phi)=(M+H-Q-\Omega) \phi$ is an "error" function which we shall estimate. Let $u$ be the potential of $e(\phi)$ (obtained by convolving with the Newton kernel). Then $u$ satisfies $A u=e(\phi)$ and the function $f=\phi+u$ is harmonic. Applying Lemma 7.4 to $f$ leads to the inequality

$$
\int_{B}\left|\nabla_{r} \phi\right|^{2} \leqq 2 \int_{B}\left|\nabla_{\theta} \phi\right|^{2}+4 \int_{B}|\nabla u|^{2} .
$$

There are standard estimates on the last integral. In particular, (Morrey, Theorem 3.7.3b) if $e(\phi) \in L_{4 / 3}(B)$ then $\|\nabla u\|_{02}^{2} \leqq c\|e(\phi)\|_{0,4 / 3}^{2}$. This can be estimated by Hölder's inequality and Lemma 7.2 :

$$
\begin{aligned}
\|e(\phi)\|_{0,4 / 3}^{2} & \leqq\left\|(M+H-\Omega) \phi+\left.2|\omega| \cdot|| \nabla \phi|+| \omega\right|^{2} \cdot|\phi|\right\|_{0,4 / 3}^{2} \\
& \leqq c\|\phi\|_{04}^{2}\left(\|M\|_{02}^{2}+a^{2}\|\phi\|_{04}^{4}+\|\Omega\|_{02}^{2}+\|\omega\|_{04}^{4}\right)+2\|\omega\|_{04}^{2}\|\nabla \phi\|_{02}^{2} \\
& \leqq c \varepsilon^{2}\|M\|_{02}^{2}+c \varepsilon^{2} E^{2}
\end{aligned}
$$

when $\varepsilon$ is sufficiently small. The lemma follows.

Theorem 7.6. Let $(\phi, \Omega)$ be a $C^{\infty}$ solution of the coupled field equations on $B_{2}-\{0\}$. Assume that the energy $\varepsilon$ is sufficiently small. Then for some constant $c$

$$
\left.\int_{B_{1}}|| \nabla \phi\right|^{2}+|\phi|^{2}-c \varepsilon E^{2}-c \varepsilon \int_{B_{1}}|M|^{2} \leqq\left. c \int_{S_{1}}|| \nabla \phi\right|^{2}+|\phi|^{2} .
$$

Proof. We are going to subtract from $\phi$ a radially symmetric solution to $\Delta \psi=0$ on each annulus $A_{n}$, and integrate by parts. Let $\bar{\phi}(r)$ be the average value of $\phi$ on $S_{r}$ and let $\psi_{n}$ be the function on $A_{n}$ linear in $1 / r^{2}$ with values $\psi_{n}=\bar{\phi}$ on $\partial A_{n}$. Then $\psi_{n}$ is continuous and radially symmetric and $\Delta \psi_{n}=0$. Furthermore, because $0 \leqq|\nabla(\phi-\psi)|^{2}=|\nabla \phi|^{2}+|\nabla \psi|^{2}-2 \operatorname{Re}\langle\nabla \phi, \nabla \psi\rangle$ and

$$
\int_{A_{n}}\langle\nabla \phi, \nabla \psi\rangle=\int_{\partial A_{n}}\langle\phi, \nabla \psi\rangle=\int_{\partial A_{n}}\langle\psi, \nabla \psi\rangle=\int_{A_{n}}|\nabla \psi|^{2}
$$

on each $A_{w}$ the function $\psi=\left\{\psi_{n}\right\}$ satisfies

$$
\int_{B_{1}}|\nabla \psi|^{2} \leqq \int_{B_{1}}|\nabla \phi|^{2}
$$

Set $f=\phi-\psi$. Then $\int_{S_{n}} f=0$ and by the Poincaré and Kato inequalities

$$
\int_{S_{1}}|f|^{2} \leqq \lambda \int_{S_{1}}|\bar{d}| \|^{2} \leqq\left.\lambda \int_{S_{1}}|| V_{\theta} f\right|^{2}
$$

where $\nabla_{\theta}$ is the covariant derivative on $S_{1}$ and $\lambda^{-1}$ is the first non-zero eigenvalue of the Laplacian on $S_{1}$.

With these facts, we shall examine the integration by parts formula

$$
\int_{A_{n}}\langle|\nabla f,| \nabla \phi\rangle-\left\langle f,\left|\nabla^{*}\right| \nabla \phi\right\rangle=\int_{\partial A_{n}}\left\langle f, \mid \nabla_{r} \phi\right\rangle .
$$


The first step is to sum this equation on $n$ to get an integral over $B_{1}$. For this it is necessary to check that the intermediate boundary terms cancel and that the inside boundary term vanishes as $n \rightarrow \infty$.

The intermediate boundary terms will cancel if $\left\langle f, \mid \nabla_{r} \phi\right\rangle$ is continuous across each $S_{n}$. But $\psi$ is continuous and - because the gauges from $A_{n}$ and $A_{n+1}$ agree on $S_{n}-\phi, \mid \nabla \phi$ and hence $\left\langle f, \mid \nabla_{r} \phi\right\rangle$ are continuous in the broken harmonic gauge. As for the inside boundary term, the bounds $|\mathbb{V} \phi(x)| \leqq c E(2|x|) \cdot|x|^{-2}$ and $|\phi(x)| \leqq c E(2|x|)$ $\cdot|x|^{-1}$ from Corollary 6.4 give

$$
\left|\int_{S_{n}}\left\langle f, \mid \nabla_{r} \phi\right\rangle\right| \leqq \sup (|\phi|+|\bar{\phi}|) \cdot c E(2 r) r^{-2} \cdot \operatorname{Vol} S_{n} \leqq c E^{2}(2 r) \quad r=2^{-n} .
$$

But $\lim _{r \rightarrow 0} E(r)=0$, so this vanishes as $n \rightarrow \infty$.

After summing on $n$ and using the field equations, (7.4) reads

$$
\int_{B_{1}}\langle|\nabla f,| \nabla \phi\rangle-\langle f,(M-\Omega-H) \phi\rangle=\int_{S_{1}}\left\langle f, \mid \nabla_{r} \phi\right\rangle .
$$

The sphere integral can be estimated by first using (7.3)

$$
\int_{S}\left\langle f, \mid \nabla_{r} \phi\right\rangle \leqq \frac{1}{2} \int_{S}|f|^{2}+\left.|| \nabla_{r} \phi\right|^{2} \leqq\left.\frac{1+\lambda}{2} \int_{S}|| \nabla_{\theta} f\right|^{2}+\left.|| \nabla_{r} \phi\right|^{2},
$$

and writing $\left.|| \nabla_{\theta} f\right|^{2}=\left|\nabla_{\theta} \phi\right|^{2}-2 \operatorname{Re}\left\langle\left|\nabla_{\theta} \phi,\right| \nabla_{\theta} \psi\right\rangle+\left.|| \nabla_{\theta} \psi\right|^{2}$. Note that $0=\nabla_{\theta} \psi=\mid \nabla_{\theta} \psi$ $-\omega_{\theta} \psi$ and $\bar{d}^{*} \omega_{\theta}=0$ by Theorem $7.1 \mathrm{c}$ so, integrating by parts,

Hence

$$
\int_{S}\left\langle\mid \nabla_{\theta} \phi, \omega_{\theta} \psi\right\rangle=-\int_{S}\left\langle\phi, \omega_{\theta} \mid \nabla \psi\right\rangle=\int_{S}\left\langle\omega_{\theta} \phi, \omega_{\theta} \psi\right\rangle .
$$

$$
\begin{aligned}
\left.\int_{S}|| \nabla_{\theta} f\right|^{2}-\left.|| \nabla_{\theta} \phi\right|^{2} & =\int_{S}\left|\omega_{\theta} \psi\right|^{2}-2 \operatorname{Re}\left\langle\omega_{\theta} \phi, \omega_{\theta} \psi\right\rangle \leqq \int_{S}\left|\omega_{\theta} f\right|^{2} \\
& \leqq\left\|\omega_{\theta}\right\|_{\infty}^{2} \int_{S}|f|^{2} \leqq\left.\lambda b^{2} \varepsilon^{2} \int|| \nabla_{\theta} f\right|^{2}
\end{aligned}
$$

by Theorem 7.1e and (7.3). When $\varepsilon$ is sufficiently small this gives

$$
\left.\int_{S}|| \nabla_{\theta} f\right|^{2} \leqq\left. 2 \int_{S}|| \nabla_{\theta} \phi\right|^{2}
$$

and (7.6) becomes

$$
\int_{S}\left\langle f, \mid \nabla_{r} \phi\right\rangle \leqq\left.(1+\lambda) \int_{S}|| \nabla \phi\right|^{2} .
$$

To estimate the left-hand side of (7.5), we write $\langle\nabla f, \mid \nabla \psi\rangle=\langle\nabla f, \nabla \phi\rangle$ $+\langle\omega f, \mid \nabla \phi\rangle+\langle\nabla f, \omega \phi\rangle$ and bound $\langle\nabla f, \nabla \phi\rangle=|\nabla \phi|^{2}-\left\langle\nabla_{r} \psi, \nabla_{r} \phi\right\rangle$ below using (7.2) and Lemmas 7.5 and 7.3

$$
\begin{aligned}
\int_{B_{1}}\langle\nabla f, \nabla \phi\rangle & \geqq \int_{B_{1}}\left|\nabla_{\theta} \phi\right|^{2}+\left|\nabla_{r} \phi\right|^{2}-\left(\left|\nabla_{r} \phi\right|^{2}+\frac{1}{4}\left|\nabla_{r} \psi\right|^{2}\right) \\
& \geqq \int_{B_{1}} \frac{1}{3}|\nabla \phi|^{2}-c \varepsilon^{2} E^{2}-c \varepsilon^{2}\|M\|_{02}^{2}-\frac{1}{4}|\nabla \phi|^{2} \\
& \geqq \frac{1}{12} \int_{B_{1}}|\nabla \phi|^{2}-c \varepsilon^{2}\left(E^{2}+\|M\|_{02}^{2}\right) .
\end{aligned}
$$


Using Lemma 7.2 we also have

$$
\text { (i) } \begin{aligned}
\|\langle\omega f, \mid \nabla \phi\rangle\|_{01} & \leqq\|\omega\|_{04}^{2}+\|\nabla \phi\|_{02}^{2} \cdot\|f\|_{04}^{2} \leqq c \varepsilon^{2} E^{2}+c \varepsilon^{2}\|f\|_{04}^{2}, \\
\text { (ii) }\|\langle\nabla f, \omega \phi\rangle\|_{01} & \leqq\|\omega\|_{04}^{2}+\|\phi\|_{04}^{2} \cdot\|\nabla \phi\|_{02}^{2} \leqq c \varepsilon^{2} E^{2}, \\
\text { (iii) }\|\langle f,(M-\Omega-H) \phi\rangle\|_{01} & \leqq\|\phi\|_{04}\left(\|M\|_{02}^{2}+\|\Omega\|_{02}^{2}+a\|\phi\|_{04}^{2}+\|f\|_{04}^{2}\right) \\
& \leqq c \varepsilon\left(E^{2}+\|M\|_{02}^{2}+a\|\phi\|_{04}^{2}+\|f\|_{04}^{2}\right) .
\end{aligned}
$$

Combining (7.5), (7.8), (7.9) and these estimates yields

$$
\left.\int_{B_{1}}\left\|\left.\nabla \phi\right|^{2}-c \varepsilon\left(E^{2}+\|M\|_{02}^{2}\right)-c \varepsilon\left(\|\phi\|_{04}^{2}+\|f\|_{04}^{2}\right) \leqq c \int_{S_{1}}\right\| \nabla \phi\right|^{2}
$$

It remains to estimate $\|f\|_{04}^{2}$ and $\|\phi\|_{04}^{2}$. For this we use the Sobolev-type inequality (see Morrey, p. 82)

$$
\int_{B_{1}}|\phi|^{2} \leqq \mathcal{C}_{0}\left[\left.\int_{B_{1}}|| \nabla \phi\right|^{2}+\int_{S_{1}}|\phi|^{2}\right]
$$

which can be combined with the usual Sobolev inequality to give

$$
\|\phi\|_{04}^{2} \leqq c\left[\left.\int_{B_{1}}|| \nabla \phi\right|^{2}+\int_{S_{1}}|\phi|^{2}\right] .
$$

We can also apply (7.12) to $f$. Note that $\mid \nabla f=\nabla f+\omega f$ satisfies

$$
\|\nabla f\|_{02}^{2} \leqq 2\|V f\|_{02}^{2}+2\|\omega\|_{04}^{2} \cdot\|f\|_{04}^{2} \text {, }
$$

that $\|\omega\|_{04}^{2} \leqq c \varepsilon^{2}$ by Lemma 7.2a, and that

$$
\|\nabla f\|_{02}^{2} \leqq 2\|\nabla \phi\|_{02}^{2} \leqq c\left(1+\varepsilon^{2}\right) E^{2}
$$

by (7.2) and Lemma 7.3. The sphere integral is bounded by (7.3) and (7.7). It follows that, for $\varepsilon$ sufficiently small,

$$
\|f\|_{04}^{2} \leqq c E^{2}+c \int_{S_{1}} \|\left. P \phi\right|^{2}
$$

Finally, multiply (7.10) by $1+c_{0}$ and add to (7.11), incorporating (7.12) and (7.13). The theorem follows when $\varepsilon$ is sufficiently small.

We next derive an analogous estimate for the curvature field $\Omega$. This theorem was obtained by Uhlenbeck [19] when $\Omega$ is a Yang-Mills field. We repeat her proof, adding a current term.

Theorem 7.7. Suppose that $\Omega$ satisfies $D^{*} \Omega=J$ on $B_{2}-\{0\}$, where $J$ is the fermion or boson current. Then for $\varepsilon$ sufficiently small there is a constant $c$ such that

$$
\int_{B_{1}}|\Omega|^{2}-c \varepsilon E^{2} \leqq c \int_{S_{1}}|\Omega|^{2}
$$

Proof. Again, integrate by parts in the broken harmonic gauge and estimate the extraneous terms. Since $D \omega=d \omega+[\omega, \omega]=\Omega+\frac{1}{2}[\omega, \omega]$, the differential of the R-valued 3-form $\omega \wedge * \Omega$ is

$$
\begin{aligned}
d(\omega \wedge * \Omega) & =D \omega \wedge * \Omega-\omega \wedge D(* \Omega) \\
& =\Omega \wedge * \Omega+\frac{1}{2}[\omega, \omega] \wedge * \Omega-\omega \wedge * D^{*} \Omega \\
& =\left[|\Omega|^{2}+\frac{1}{2}\langle[\omega, \omega], \Omega\rangle-\left\langle\omega, D^{*} \Omega\right\rangle\right] \cdot \text { volume form }
\end{aligned}
$$


From the field equations $\left\langle\omega, D^{*} \Omega\right\rangle=J(\omega)$, so on the annulus $A_{n}$

$$
\int_{\partial A_{n}} \omega \wedge * \Omega=\int_{A_{n}}|\Omega|^{2}+\frac{1}{2}\langle[\omega, \omega], \Omega\rangle-J(\omega) .
$$

Because $\partial A_{n}$ is spherical only the tangential components $\omega_{\theta} \wedge(* \Omega)_{\theta}$ contribute to the boundary integral. But the components of $(* \Omega)_{0}$ are the components of $\Omega$ with a radial part, so we can replace $(* \Omega)_{\theta}$ by $\Omega_{r}=\sum \Omega_{r i} d r \wedge e^{i}$.

Now sum on $n$. The intermediate boundary terms cancel as before. The inside boundary term can be estimated using Corollary 6.4 and Theorem 7.1e

$$
\int_{S_{n}}\left\langle\omega_{\theta}, \Omega_{r}\right\rangle \leqq\left\|\Omega^{r}\right\|_{\infty} \cdot\left\|\omega^{n}\right\|_{\infty} \cdot \operatorname{Vol} S_{n} \leqq c r^{-2} E(2 r) \cdot b r^{-1} \varepsilon \cdot c r^{3} \leqq c E(2 r),
$$

which vanishes when $r=2^{-n} \rightarrow 0$. This gives

$$
\int_{B_{1}}|\Omega|^{2}+\frac{1}{2}\langle[\omega, \omega], \Omega\rangle-J(\omega)=\int_{S_{1}}\left\langle\omega_{\theta}, \Omega_{r}\right\rangle .
$$

Now by Lemma 7.2 and Theorem 7.1f we have

$$
\int_{B}\langle[\omega, \omega], \Omega\rangle \leqq\|\omega\|_{04}^{2} \cdot\|\Omega\|_{02} \leqq c \varepsilon E^{2}
$$

and can bound the fermion and boson currents, respectively by

$$
\begin{aligned}
& \left.\|J(\omega)\|_{01}=\| \sum\left\langle\phi, e^{i} \cdot \varrho\left(e_{i}\right\lrcorner \omega\right) \phi\right\rangle\left\|_{01} \leqq\right\| \omega\left\|_{02} \cdot\right\| \phi\left\|_{04}^{2} \leqq\right\| \Omega\left\|_{02}^{2} \cdot\right\| \phi \|_{04}^{2} \leqq c \varepsilon E^{2} \\
& \left.\|J(\omega)\|_{01}=\| \sum \operatorname{Re}\left\langle\nabla_{i} \phi, \varrho\left(e_{i}\right\lrcorner \omega\right) \phi\right\rangle\left\|_{01} \leqq\right\| \phi \|_{04}\left(\|V \phi\|_{02}^{2}+\|\omega\|_{04}^{2}\right) \leqq c \varepsilon E^{2} .
\end{aligned}
$$

Also, $\int_{S}\left|\omega_{\theta}\right|^{2} \leqq c \int_{S}\left|\Omega_{\theta \theta}\right|^{2}$ from the proof of Lemma 7.2, so

$$
\int_{S}\left\langle\omega_{\theta}, \Omega_{r}\right\rangle \leqq c \int_{S}\left|\Omega_{\theta \theta}\right|^{2}+\left|\Omega_{r}\right|^{2} \leqq c \int_{S}|\Omega|^{2} .
$$

The theorem now follows from (7.14).

Finally, we combine the previous two theorems and make explicit how these estimates depend on the metric within the conformal class.

Theorem 7.8. Let $(\phi, \Omega)$ be a solution to the coupled fermion or boson field equations with locally finite energy at a singularity $p \in M$. Assume that the mass coefficient $m$ satisfies the hypotheses of Theorem 6.3. Then for all $r \leqq r_{0}$ the energy in the ball of radius $r$ around $p$ satisfies

$$
E(r) \leqq c r^{\delta} \text { for some } \delta>0 .
$$

Proof. Rescale and fix a metric $g_{0}$ in which $(\phi, \Omega)$ is defined on $B_{2}-\{0\}$ and $\varepsilon$ is small enough for the previous theorems to hold. Let $B(\tau, r)$ be the ball of radius $r$ in the metric $g=\tau^{-4} g_{0}$ and let $E(\tau, r)$ be the integral (3.5) of the energy density over $B(\tau, r)$. Then $E(\tau, r)=E\left(1, \tau^{2} r\right)$ and the function $E(\tau)=E(\tau, 1)$ satisfies

$$
\left.\frac{d}{d \tau} E^{2}(\tau)\right|_{\tau=1}=\left.\frac{d}{d \tau} E^{2}\left(1, \tau^{2}\right)\right|_{\tau=1}=\left.2 \frac{d}{d r} E^{2}(r)\right|_{r=1} .
$$

The expression $\left.\frac{d}{d r} E^{2}(r)\right|_{r=1}$ is the integral of the energy density over the unit sphere. 
Adding the inequalities of Theorems 7.6 and 7.7 gives

$$
E^{2}(\tau)-c \varepsilon \cdot \int_{B(\tau, 1)}|M|^{2} \leqq c \tau^{2}\left[E^{2}(\tau)\right]^{\prime}
$$

when $\tau=1$. But this equation is conformally invariant ( $M$ has weight 2 and the sphere integral has weight -1 ) except for the factor $\varepsilon$, which decreases as $\tau$ decreases. Hence (7.15) holds for all $\tau \leqq 1$ with $\varepsilon=E(1,1)$ fixed.

The hypothesis $M \in L_{1, p}\left(B_{1}\right)$ for some $p>2$ implies, by Hölder's inequality, $\underset{B(1, r)}{ }|\mathrm{M}|^{2} \leqq \mathrm{c}_{1} \mathrm{r}^{\delta}$ for some $\delta>0$. Since $M$ has conformal weight 2 we have

$$
\int_{B(\tau, 1)}|M|^{2}=\int_{B(1, \tau)}|M|^{2} \leqq c_{1} \tau^{\delta} .
$$

Substituting into (7.15) yields the differential inequality $E^{2}(\tau)-c \tau^{2}\left(E^{2}(\tau)\right)^{\prime} \leqq c_{1} \tau^{\delta}$, $\tau \leqq 1$, and integrating this gives (even when $M=c_{1}=0$ )

$$
E(1, \tau)=E(\tau) \leqq c \tau^{\delta^{\prime}}
$$

for some $c$ and $\delta^{\prime}>0$.

\section{Removable Singularities}

The regularity theorems of Sect. 5 and the energy growth rate established in Sect. 7 together yield a theorem on the removability of singularities.

Theorem 8.1. Let $(\phi, \Omega)$ be a solution to the coupled field equations (2.5), (2.6) with finite energy in a neighborhood $D$ of $p \in M$. Assume that $(\phi, \Omega)$ is $C^{\infty}$ in $D-\{p\}$ and that the mass coefficient $m$ satisfies

(i) $m \in L_{2, p}$ (D) for some $p>2$,

(ii) $m^{2} \in L_{1, p}(D)$ for some $p>2$,

in the fermion and boson cases, respectively. Then there exists a continuous trivialization of the principal bundle over $D$ in which the connection and the field $\phi$ extend over $p$ to $C^{\infty}$ solutions of the field equations.

Proof. By rescaling we may assume that $D-\{p\}=B_{2}-\{0\}$ and that a broken harmonic gauge exists. Theorem 7.8 then implies that $F \in L_{p}$ for some $p>2$, so $\phi \in L_{1, p}$ and $\Omega \in L_{p}$. Furthermore, since $d^{*} \omega=0$ in the broken harmonic gauge,

$$
|\nabla \omega|=|d \omega|=\left|\Omega-\frac{1}{2}[\omega, \omega]\right| \leqq|\Omega|+|\omega|^{2}
$$

and hence $\omega \in L_{1, p}$ (using Lemma 7.2a). Regularity now follows from Theorem 5.3 .

Finally, we will use Theorem 8.1 to show how solutions to the coupled fermion or boson equations defined on $\mathbb{R}^{4}$ extend to solutions on $S^{4}$.

Suppose that $(\phi, \Omega)$ is a $C^{\infty}$ solution to the coupled field equations on $\mathbb{R}^{4}$. Let $\sigma: S^{4} \rightarrow \mathbb{R}^{4}$ be the stereographic projection from the north pole $p$. Because $\sigma$ is a conformal transformation and the field equations are conformally invariant, the pulled back field $\left(\sigma^{*} \phi, \sigma^{*} \Omega\right)$ is a $C^{\infty}$ solution on $S^{4}-\{p\}$. Theorem 8.1 will now show that the singularity at the north pole is removable provided that the energy is 
finite and that the condition on $m$ is valid. To state this condition, it is convenient to follow the stereographic projection $\sigma$ by the stereographic projection $\sigma^{\prime}$ from the south pole. The composition $\sigma^{\prime} \sigma$ is the conformal inversion $f: \mathbb{R}^{4} \rightarrow \mathbb{R}^{4}$ by $f(x)=x /|x|^{2}$ which transports the singularity to the origin of $\mathbb{R}^{4}$. The differential of $f$ is $f_{*}(x) \cdot X=|x|^{2} \cdot X$ for $X \in T_{x} \mathbb{R}^{4}=\mathbb{R}^{4}$. A quantity $\xi$ of conformal weight $w$ then satisfies $\left(f^{*} \xi\right)(x)=|x|^{-2 w} \xi\left(\frac{x}{|x|^{2}}\right)$. We can then translate the hypotheses of Theorem 8.1 into hypotheses on the original field on $\mathbb{R}^{4}$. The conditions on $m$ become conditions involving weighted Sobolev spaces on $\mathbb{R}^{4}$ or - after the inversion - conditions at the origin. We then have:

Corollary 8.2. Let $(\phi, \Omega)$ be a $C^{\infty}$ solution to the coupled Yang-Mills equations (2.5) or $(2.6)$ on $\mathbb{R}^{4}$. Assume

1) For fermion fields $\phi$, that the energy

$$
\int_{\mathbb{R}^{4}}|\Omega|^{2}+\left.|x|^{2}|| \nabla \phi\right|^{2}+|x|^{-2}|\phi|^{2}
$$

is finite and that $|x|^{-2} m\left(\frac{x}{|x|^{2}}\right) \in L_{2, p}(p>2)$ locally at the origin.

2) For boson fields $\phi$, that the energy

$$
\int_{\mathbb{R}^{4}}|\Omega|^{2}+\left.|| \nabla \phi\right|^{2}+|x|^{-4}|\phi|^{2}
$$

is finite, that $|x|^{-4} m^{2}\left(\frac{x}{|x|^{2}}\right) \in L_{1, p}(p>2)$ locally at the origin and that $a$ and da (see (2.6)) are bounded at infinity.

Then $(\phi, \Omega)$ extends via stereographic projection to a $C^{\infty}$ solution on $S^{4}$.

Acknowledgement. I thank Professors K. Uhlenbeck and S.-T. Yau for their suggestions and encouragement.

\section{References}

1. Atiyah, M.F., Bott, R.: On the Yang-Mills equations over Riemann surfaces (preprint)

2. Atiyah, M.F., Bott, R., Patodi, V.K.: On the heat equation and the index theorem. Invent. Math. 19, 279-330 (1973)

3. Atiyah, M.F., Bott, R., Shapiro, A. Clifford modules. Topology 3, Suppl. 1, 3-38 (1964)

4. Atiyah, M.F., Hitchin, N., Singer, 1.: Self-duality in four-dimensional Riemannian geometry. Proc. R. Soc. (London) A 362, 425-461 (1978)

5. Atiyah, M.F., Singer, I.: The index of elliptic operators. III. Ann. Math. 87, 546-604 (1968)

6. Bourguignon, J.P., Lawson, H.B.: Stability and isolation phenomena for Yang-Mills fields. Commun. Math. Phys. 79, 189-230 (1981)

7. Fegan, H.: Conformally invariant first order differential operators. Q.J. Math. 27, 371-378 (1976)

8. Hawking, S., Ellis, G.: The large scale curvature of space-time. Cambridge University Press 1973

9. Hitchin, N.: Harmonic spinors. Adv. Math. 14, 1-55 (1974)

10. Hitchin, N.: Linear field equations on self-dual spaces. Proc. R. Soc. (London) A 370, 173-191 (1981)

11. Kobayashi, S., Nomizu, K.: Foundations of differential geometry, Vol. I. New York: Interscience 1963

12. Mitter, P.K., Viallet, C.M.: On the bundle of connections and the gauge orbit manifold in YangMills theory. Commun. Math. Phys. 79, 457-472 (1981) 
13. Morrey, C.B.: Multiple integrals in the calculus of variations. Berlin, Heidelberg, New York: Springer 1966

14. Palais, R.: Foundations of global non-linear analysis. New York: Benjamin 1968

15. Samelson, H.: Notes on Lie algebras. New York: Van Nostrand, Reinhold 1969

16. Singer, I.M., Thorpe, J.: The curvature of 4-dimensional Einstein spaces. In: Global analysis, Papers in honor of Kodaira, K., Spencer, D.C., Iyanaga, S. (eds.). Princeton NS: Princeton University Press 1969,1969 , pp. 355-365

17. Sternberg, S.: On the role of field theories in our physical conception of geometry. In: Differential geometrical methods in mathematical physics, Vol. II. Bleuler, Petry, Reetz (eds.). Lecture Notes in Mathematics, Vol. 1b, pp. 1-55. Berlin, Heidelberg, New York: Springer 1978

18. Stredder, P.: Natural differential operators on riemannian manifolds and representations of the orthogonal and special orthogonal groups. J. Diff. Geol, 10, 657-660 (1975)

19. Uhlenbeck, K.: Removable singularities in Yang-Mills fields. Commun. Math. Phys. 83, 11-30 (1982)

20. Uhlenbeck, K. Connections with $L^{p}$ bounds on curvature. Commun. Math. Phys. 83, 31-42 (1982)

Communicated by A. Jaffe

Received December 2, 1981; in revised form March 8, 1982 